\title{
Littoral Sedimentary Processes on Kauai, A Subtropical High Island ${ }^{1}$
}

\author{
D. L. INMAN, ${ }^{2}$ W. R. GAYMAN, ${ }^{2}$ and D. C. $\mathrm{COX}^{3}$
}

\begin{abstract}
Beach and shallow water sand samples from the island of Kauai, Hawaiian Islands, were studied to ascertain the effects of climate on the supply of sediment, and of wave action on the dispersal and transport of sand along the shores of this circular island. The littoral sediments of the island are made up of two components: biogenous material, such as shell, coral, and foraminiferal sands, formed near the shoreline; and terrigenous material, consisting mostly of volcanic mineral and rock fragments brought to the beach by rivers.

The northeast trade winds play a dominant part in the climatic and oceanographic processes affecting the island. On the windward side of Kauai, the annual rainfall ranges from 30 inches near the coast to over 460 inches at higher elevations; on the leeward side, annual rainfall is less than 20 inches. This large variation in rainfall results in a climatic range from tropic-humid to semi-arid. Rivers draining the wet, windward portion of the island carry relatively little sand, and the shoreline on this side is characterized by intermittent fringing reefs and beaches of calcareous sand. A detailed study of several reefs on the windward side of the island shows that each pair of fringing reefs, divided by a relatively deep inlet, constitute separate cells for the circulation of water and distribution of sediments.

The highest concentrations of volcanic sand occur on the lee side of the island, where streams draining the semi-arid region enter the coast. The concentration of volcanic material in the beach sands decreases logarithmically with distance along the coast from the rivers.
\end{abstract}

KAUAI is the next to westernmost of the windward islands of the Hawaiian Archipelago (Fig. 1 ). It is roughly circular in shape, with a diameter of about 25 nautical miles (Fig. 2). The island is volcanic and has a central plateau whose eastern rim exceeds 5,000 ft in elevation. This mile-high rim intercepts the trade winds, causing moderate to extreme precipitation on the windward slope and producing a rainshadow desert along portions of the leeward coast.

\footnotetext{
${ }^{1}$ Published as Contribution No. 24 of the Hawaii Institute of Geophysics and, with the approval of the Director, as Paper Number 88 in the Journal Series of the Experiment Station, Hawaiian Sugar Planters' Association. Manuscript received November 22, 1961.

${ }^{2}$ Scripps Institution of Oceanography of the University of California, La Jolla, California.

${ }^{3}$ Hawaii Institute of Geophysics, University of $\mathrm{Ha}$ waii; formerly Experiment Station, Hawaiian Sugar Planters' Association.
}

The contrast between windward and leeward sides of the island in terms of climate and source of sediment suggested that this island was an ideal locality to study the relation between nearshore sedimentation and the source and type of sediment. It was found that the windward streams, although high in runoff, contributed very little sand-size material to the nearshore areas. On this side, the coastal environment is characterized by fringing coral reefs, with protected beaches composed of calcareous sand derived from shells, reef detritus, and foraminifera. Kapaa Reef had been investigated by Kohn and Helfrich (1957) and, since it seemed typical of the windward reefs, it was selected for more detailed study.

Consistently high concentrations of terrigenous material were found on only a few of the leeward beaches. These deposits were particularly conspicuous near the mouth of the Wai- 


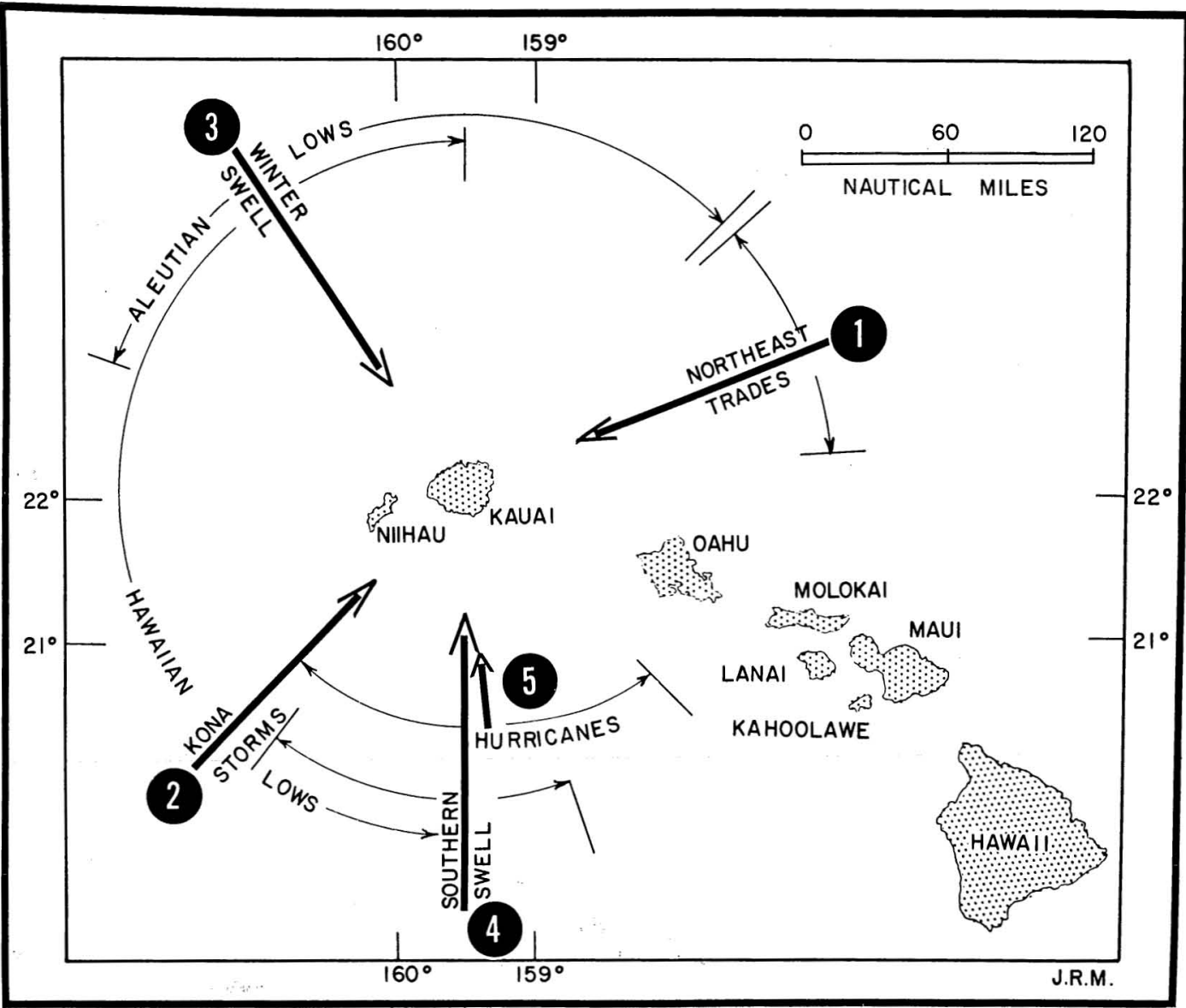

FIG. 1. Index chart and exposure to waves and winds. Heavy arrows indicate directions of approach of the five major wave types which affect the island.

mea River, which drains the major canyon on the island and is in an area of relatively light rainfall. The most typical of the lee beaches investigated lay along the shores of the Mana Coastal Plain.

During the study, it was observed that beach and dune rock is especially well developed along portions of the Kauai coast. This was the subject of a special investigation by Emery and Cox (1956). Some additional observations are discussed under the section on sediments.

\section{PHYSIOGRAPHY}

Kauai is one of a chain of volcanic islands in the Hawaiian Archipelago. It formed as a shield volcano built up from the deep floor of the $\mathrm{Pa}$ - cific Ocean by thin flows of basaltic lava. The original shield collapsed to form a broad caldera, which subsequently filled with thicker lava flows and talus deposits. The caldera is now almost obscured by faulting, extensive erosion, and stream cutting. Ninety $\%$ of the flows are composed of normal olivine basalt, while the remaining rock is a basalt either very rich (picrite-basalt) or very poor in olivine. A comprehensive and up-to-date review of the geology of Kauai is given by Macdonald, Davis, and Cox (1960).

The ocean floor around Kauai is characterized by a number of sloping terraces which are terminated on their seaward side by a marked increase in slope. Although the depths of the breaks in slopes at the outer edges of the ter- 
races range on various sides of the island from $30 \mathrm{ft}$ to $500 \mathrm{ft}$, their grouping suggests that terracing occurred at about four separate levels. The shoalest and widest terrace commonly extends to depths of 40 to $90 \mathrm{ft}$; others extend from about $150 \mathrm{ft}$ to between 240 to $350 \mathrm{ft}$; and for the deepest, to about $450 \mathrm{ft}$. The latter depth presumably marks the outer edge of a wave-cut terrace formed during a still stand near the lowest Wisconsin Sea Level. Generally, the $450-\mathrm{ft}$ contour ranges from 1 to 2 miles from shore, although it extends to 4 miles off the west coast.

The most gently sloping part of the insular shelf, that part shoaler than $90 \mathrm{ft}$, is frequently characterized by many apparently closed depressions, generally ranging from 10 to $30 \mathrm{ft}$ deep. These are most common on the north coast, although a very large depression occurs on the west coast off Makaha Point (Fig. 2). The configuration of the submarine ridge surrounding this depression suggests that it may be a relic coastal sand dune, formed at a lower stand of the sea, or possibly a relic barrier beach or reef. ${ }^{4}$

\footnotetext{
${ }^{4}$ Observations made while diving on this reef during September 1962 subsequent to submission of this manuscript show the reef to consist of a framework of coral. It is believed that it represents a barrier reef formed during a previous lower stand of sea, and the name "Kaheko" is proposed for the reef because of its presumed great age.
}

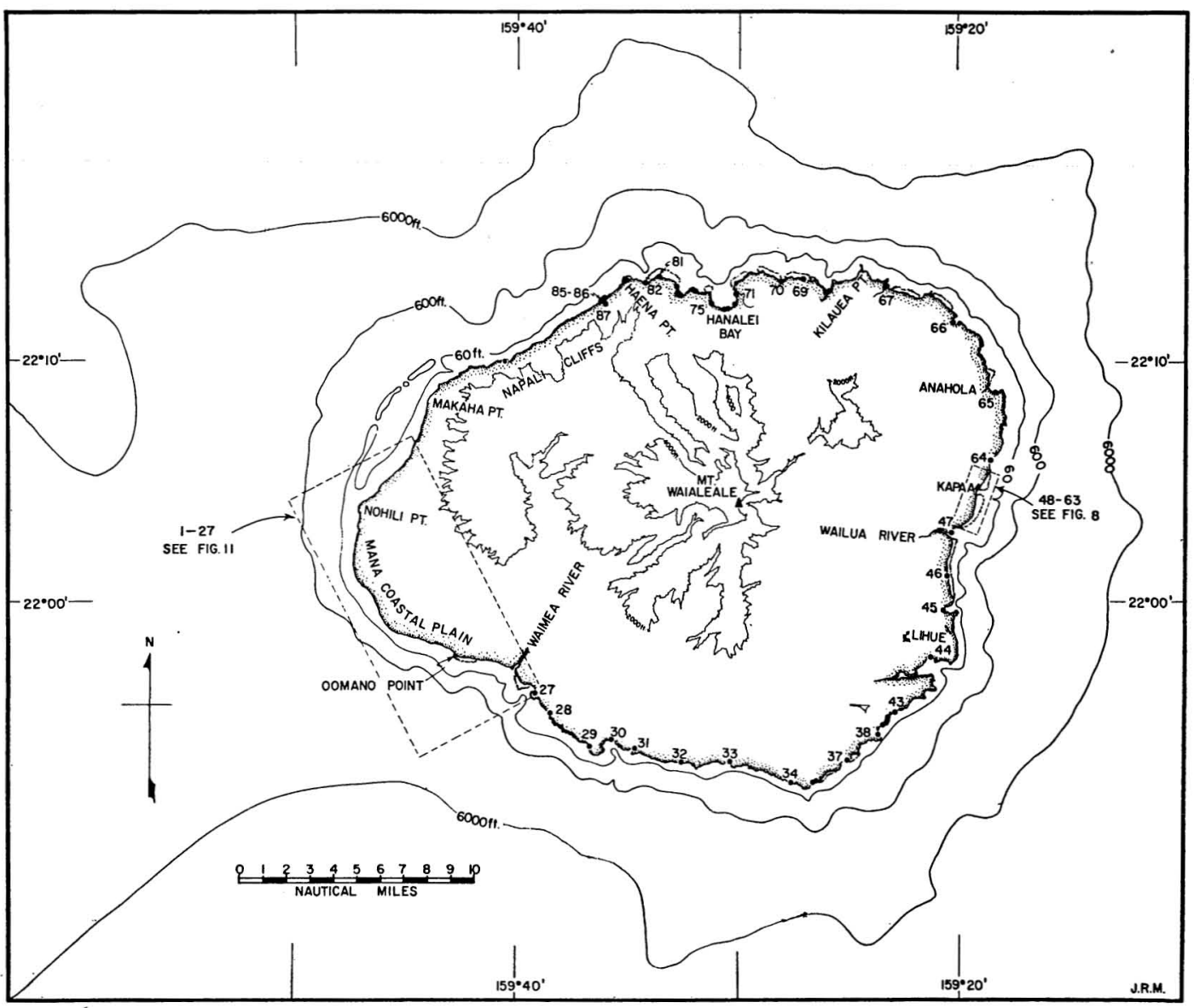

FIG. 2. Topography, bathymetry, and sample locations. Numbers along coastline refer to sample designations listed in text and in Table 2. Intermittent jagged line along shore shows location of major reefs. 


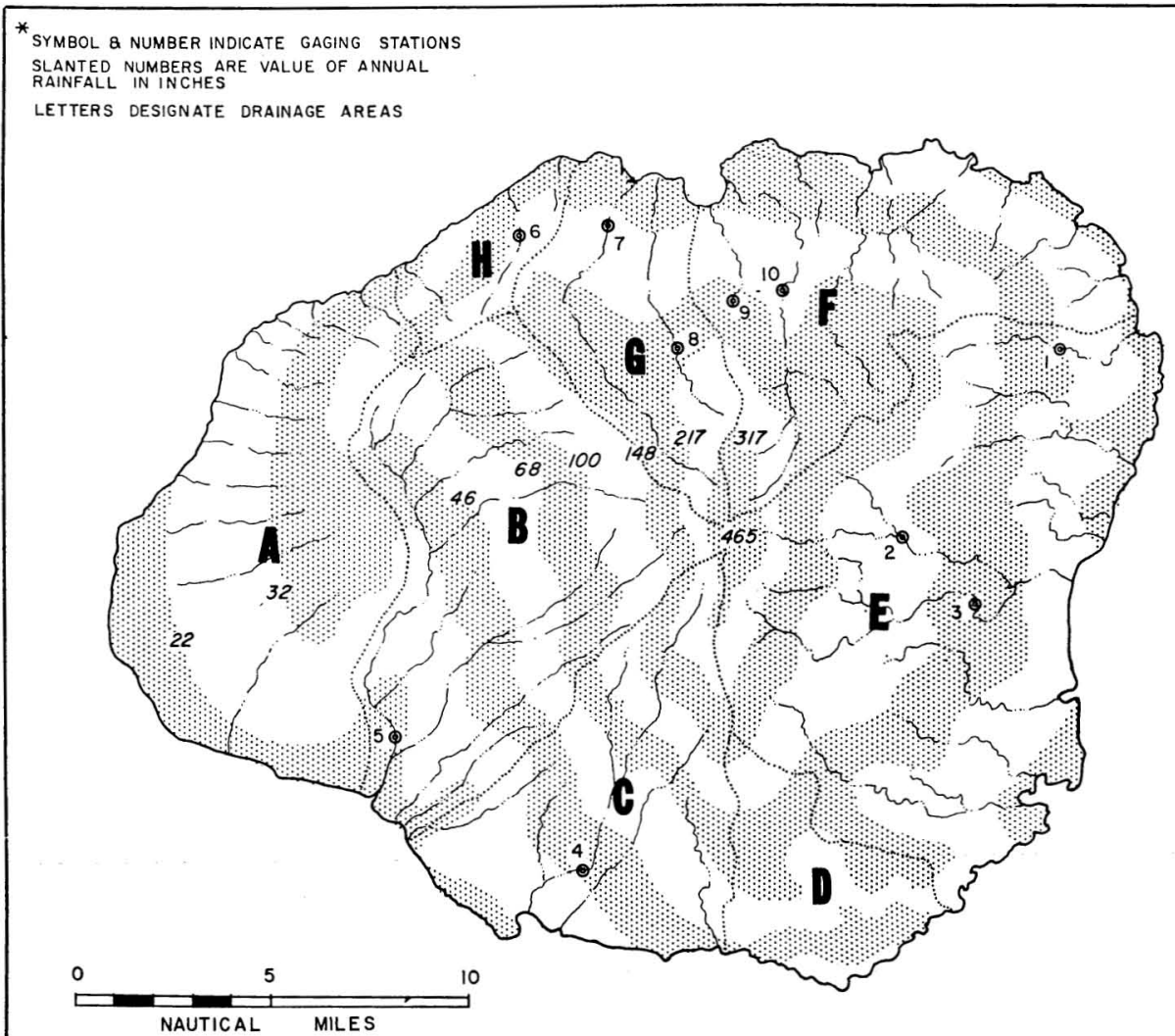

FIG. 3. Rainfall and drainage basins on Kauai. Dotted lines indicate outline of drainage areas. Numbers and letters, designating stream gaging stations and drainage areas, respectively, are referred to in Table 1 . Note area of lowest rainfall along western coast. Isohytel lines from the Meteorology Department, Pineapple Research Institute, and the Experiment Station, Hawaiian Sugar Planters' Association, 1948.

There are at least five well-developed sea valleys which cut the shallower portions of the insular shelf. All of these, except the one off Hanalei Bay, appear to terminate above the 450 -ft contour.

\section{CLIMATE}

The annual precipitation varies from an average of about 50 inches, on the windward (northeastern) shore, to over 450 inches, at the summit of Mt. Waialeale, and decreases to less than 20 inches in the rain-shadow produced on the extreme lee side of the island. Vegetation varies from tropical rain forest on the windward side, to arid on the southwestern slopes. Figure 3 shows the drainage pattern, the outlines of the major drainage basins, and the contour lines of mean annual rainfall, or isohyets, for the island, as well as the location of several streamflow gaging stations.

Langbein and Schumm (1958) have shown that the sediment yield from a drainage basin varies as a function of climate, and that the maximum yield occurs for an effective precipitation of about 12 inches. The effective precipitation $^{5}$ is obtained from the actual precipitation by correcting for evapotranspiration losses to a standard temperature of $50 \mathrm{~F}$. Sediment yield is less for precipitation either greater or less than

\footnotetext{
${ }^{5}$ Langbein and Schumm (1958) define "effective precipitation" as the amount of precipitation that would be required at a mean temperature of $50 \mathrm{~F}$ to produce the actual annual runoff from the basin.
} 
this optimum value of about 12 inches (Fig. 4). Although sediment yield is influenced by vegetation, temperature, rainfall characteristics, and topography, a rough approximation for sediment yield can be obtained from the effective precipitation.

Data indicative of the differences between the climates of the various drainage basins on Kauai and of the supplies of sediments in those basins are given in Table 1. These data include: (1) a rough calculation of the mean annual rainfall for the various drainage areas; (2) effective precipitation calculated from rainfall; (3) runoff data for the upper portion of some of the drainage basins, obtained from gaging stations; and (4) the percentage of carbonate in the beach sand sample taken nearest the stream mouths.

In general, the table confirms that the greatest precipitation and the most runoff occur on the windward side of the island where the least amount of terrigenous sediment is found in the beach sand. The Langbein and Schumm curve (Fig. 4) shows that the potential sediment yield is appreciably higher when the effective precipitation does not exceed about 35 inches, with a maximum yield corresponding to a value of about 12 inches. The equivalent values in terms of annual rainfall on $\mathrm{Kauai}^{6}$ give the band of high sediment yield as occurring where the mean annual rainfall does not exceed 46 inches, and

${ }^{\circ}$ After correcting for evapotranspiration, following Langbein et al. (1949: fig. 2).

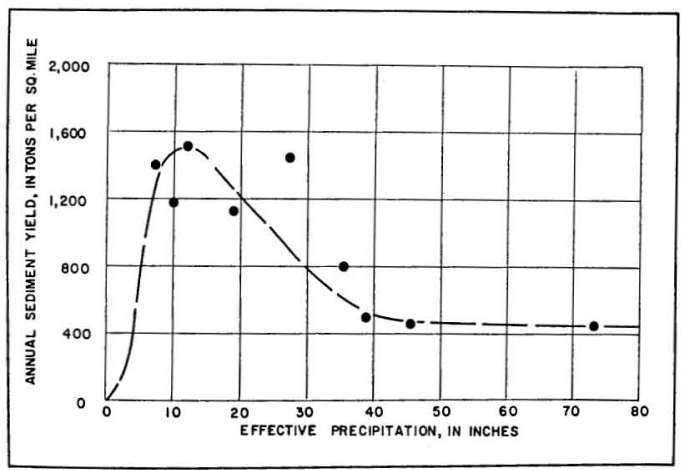

FIG. 4. Relation between effective precipitation and annual sediment yield (taken from Langbein and Schumm, 1958: fig. 3). a maximum sediment yield corresponding to a mean annual rainfall of 22 inches. Figure 3 shows that a relatively high percentage of the drainage basins on the lee side of the island fall within the range of high sediment yield, whereas the rainfall in the windward drainage basins is appreciably greater and thus produces a somewhat lower yield of sediment. The mean annual rainfall for each drainage basin and the corresponding value for the mean effective precipitation are listed in Table 1, columns 4 and 5 , respectively. The Waimea River drainage basin (Fig. 3, B), which is the principal basin on the lee side of the island, has mean values of annual rainfall and effective precipitation of 70 inches and 53 inches, respectively, which are much nearer the value of maximum sediment yield than are those for the principal windward drainage basins (Fig. 3, E, F, G). In fact, much of the Waimea drainage basin falls within the rainfall range of potentially high sediment yield. Also, the sediment yield in the Waimea basin is accentuated by the occurrence of extreme topographic relief in the low rainfall area. This combination of factors, tending to increase sediment yield, does not occur extensively in the windward basins.

The high carbonate content of the beach sand samples on the windward coast tends to confirm the difference in sediment yield between the lee and windward drainage basins (Table 1, last column). In addition, cursory inspection of river banks and river mouths indicated that not only is the yield greater on the leeward side but also that there is a significant difference in the size of the sediment: the leeward streams carry large amounts of sand-size material, whereas the windward streams carry silt and clay-size material. A predominance of silt and clay-size sediment indicates chemical weathering, a process which is enhanced in the windward basins by greater vegetation density and slower rate of removal of disintegration products.

\section{EXPOSURE TO WAVES}

Although no complete statistical wave data for spectrums and directions of waves are available for the island of Kauai, a general description of the prevailing wave conditions can be 
TABLE 1

RaInfall, Runoff, ANd Sediment Composition for DRainage Basins on KaUai

\begin{tabular}{|c|c|c|c|c|c|c|c|c|c|c|c|}
\hline & $\mathrm{DR}$ & INAGE $\mathrm{E}$ & ASIN & & & RIVER BASIN & ABOVE G & GING STA & ION & $\begin{array}{r}\text { BEAC } \\
\text { SA } \\
\text { NE } \\
\text { R }\end{array}$ & $\begin{array}{l}\text { H SAND } \\
\text { MPLE } \\
\text { AREST } \\
\text { VER }\end{array}$ \\
\hline $\begin{array}{c}\text { Desig- } \\
\text { nation* }\end{array}$ & $\begin{array}{l}\text { Area } \\
\text { (sq } \\
\text { statute } \\
\text { miles) }\end{array}$ & $\begin{array}{l}\text { Mean } \\
\text { Annual } \\
\text { Temp. } \\
\left({ }^{\circ}{ }^{\circ}\right)^{\dagger}\end{array}$ & $\begin{array}{c}\text { Mean } \\
\text { Annual } \\
\text { Rainfall } \\
\text { (inches) }\end{array}$ & $\begin{array}{l}\text { Effective } \\
\text { Precipi- } \\
\text { tation } \$ \\
\text { (inches) }\end{array}$ & $\begin{array}{l}\text { Desig- } \\
\text { nation* }\end{array}$ & River & $\begin{array}{c}\text { Drainage } \\
\text { Area } \\
\text { (sq } \\
\text { statute } \\
\text { miles) }\end{array}$ & $\begin{array}{l}\text { Runoff } \\
\text { (inches) }\end{array}$ & $\begin{array}{c}\text { Maximum } \\
\text { Inst. Flow } \\
\text { (cubic ft/ } \\
\text { sec) }\end{array}$ & No. & $\begin{array}{l}\% \text { Car- } \\
\text { bonate }\end{array}$ \\
\hline A & 86 & 70 & 30 & 18 & & & & & & & \\
\hline B & 96 & 69 & 70 & 53 & 5 & Waimea & 45.0 & 45.0 & 372 & 24 & 1.3 \\
\hline C & 64 & 69 & 76 & 55 & 4 & Hanapepe & 20.5 & 52.1 & 487 & 30 & 4.2 \\
\hline $\mathrm{D}$ & 27 & 70 & 67 & 50 & & & & & & & \\
\hline $\mathrm{E}$ & 144 & 67 & 128 & 100 & $\begin{array}{l}3 \\
2 \\
1\end{array}$ & $\begin{array}{l}\text { S. Wailua } \\
\text { N. Wailua } \\
\text { Anahola }\end{array}$ & $\begin{array}{r}22.4 \\
14.6 \\
5.5\end{array}$ & $\begin{array}{l}81.0 \\
99.8 \\
53.5\end{array}$ & $\begin{array}{r}265 \\
2045 \\
1010\end{array}$ & $\begin{array}{l}47 \\
47 \\
65\end{array}$ & $\begin{array}{l}80.0 \\
80.0 \\
90.0\end{array}$ \\
\hline $\mathrm{F}$ & 78 & 69 & 134 & 101 & $\begin{array}{r}10 \\
9\end{array}$ & $\begin{array}{l}\text { Hanalei } \\
\text { Waioli }\end{array}$ & $\begin{array}{r}19.2 \\
1.6\end{array}$ & $\begin{array}{l}196.0 \\
277.0\end{array}$ & $\begin{array}{r}1130 \\
930\end{array}$ & $\begin{array}{l}71 \\
74\end{array}$ & $\begin{array}{l}62.0 \\
76.0\end{array}$ \\
\hline G & 40 & 67 & 169 & 130 & $\begin{array}{l}8 \\
7\end{array}$ & $\begin{array}{l}\text { Lumahai } \\
\text { Wainiha }\end{array}$ & $\begin{array}{r}7.1 \\
14.7\end{array}$ & $\begin{array}{r}230.0 \\
91.9\end{array}$ & $\begin{array}{r}1090 \\
211\end{array}$ & $\begin{array}{l}76 \\
78\end{array}$ & $\begin{array}{l}62.0 \\
55.0\end{array}$ \\
\hline $\mathrm{H}$ & 14 & 67 & 93 & 70 & 6 & Hanakapiai & 2.6 & 102.0 & 1600 & 85 & 85.0 \\
\hline
\end{tabular}

* Refers to Figure 3.

$\dagger$ Adjusted for mean elevation of basin, assuming 3 F. decrease per $1000 \mathrm{ft}$ altitude. Station temperature from Macdonald, Davis, and Cox (1960: 114).

$\ddagger$ Precipitation at a mean temperature of $50 \mathrm{~F}$. required to produce the actual runoff. Runoff obtained from mean annual rainfall (extrapolation from Langbein et al., 1949: fig. 2); effective precipitation obtained from annual runoff (Langbein and Schumm, 1958: fig. 1)

$\S$ Annual river discharge divided by the drainage area. River discharge from Territorial Planning Board (1939).

obtained by generalizing from the wave summary given by the Corps of Engineers (1955: appendix B) and from specific storm descriptions, such as that of Arthur (1948). Review of these data suggests that the wave and wind regime can conveniently be classed in the following manner (Fig. 1):

1. Northeast Trades: Swell generated by the prevailing northeasterly winds. These waves are present all year, but are largest from April through November. Waves typically have periods of 5-8 sec, are 4-12 ft in height, and approach from the east-northeast.

2. Kona Storms: Waves generated by intense winds, associated with local fronts or Hawaiian lows of extra-tropical origin. The wind may vary in direction from south through west to north, and usually blows hardest from the south- west. These storms are not frequent, but occur most commonly from November through March. Waves accompanying these storms typically have periods of $8-10 \mathrm{sec}$, are $10-15 \mathrm{ft}$ high, and are most intense from the southwest.

3. Winter Swell from Aleutian and Mid-latitude Lows: Waves from this source are intermittent, and can occur throughout the year, but are most intense from October through May. Typical waves have periods of $10-17 \mathrm{sec}$, are 8-14 ft high, and may approach from the northwest, north, and northeast.

4. Soutbern Swell: Long, low waves generated by storms in the southern hemisphere, and most prevalent from June through September. Typical waves have periods of 14-22 sec, are 1-4 ft high, and approach from the southeast through the southwest. 
5. Hurricane: Waves from tropical storms, usually approaching from the southeast through southwest. They are most likely to occur in August and September, although occurrence may be between June and December.

Of the five classes of waves, those generated by the northeast trades are by far the most persistent source of wave energy for the east and northeast shores of the island. Undoubtedly these waves account for the greater abundance of calcareous reefs on the windward side of the island. Waves from Aleutian lows are probably the dominant agent responsible for recent erosion along the Napali Cliffs on the northwest coast of the island. These waves produce a southwesterly drift of littoral sediment from the Napali Cliffs towards Nohili Point and have been a major influence in the formation of the straight coast line near Barking Sands (between sample stations 1 to 7, Fig. 11). The meteorological conditions giving rise to unusually high waves of this type in January 1947 are described by Arthur (1948). Waves of this type, with breakers estimated to be $20 \mathrm{ft}$ high and periods of 14-16 sec, were observed on the north coast of Kauai on 30 November 1955.

Southern swell is the most common type of wave on the south and lee sides of the island. Although these waves are low, they are almost continuous during the summer months and produce a westerly transport of sand from the Waimea River toward the Mana Coastal Plain. Hurricanes, although infrequent, are important, and the local residents attribute some recent erosion to the combined effects of the hurricane of 1957 and the tsunami of the same year. Kauai is exposed to tsunami waves accompanying seismic activity in the vicinity of the Aleutian and $\mathrm{Ku}$ rile Trenches in the northern hemisphere, and, to a lesser degree, from tsunami waves generated off the coast of Chile in the southern hemisphere. These catastrophic waves cause considerable coastal erosion and, at times, transport large blocks of reef material inland (Shepard, Macdonald, and Cox, 1950).

\section{SEDIMENTS}

Eighty-three sand samples and five beach and dune rock samples were collected from the nearshore zone along the coasts of the island. Exami- nation of the sediment showed that it could easily be divided on the basis of origin into: (1) biogenous material derived from the skeletons of molluscs, coral, calcareous algae, and foraminifera; and (2) terrigenous material derived from erosion of the island's volcanic formations. These two sediment types were characterized by important differences in color, density, and solubility in dilute hydrochloric acid. In general, the biogenous material appeared to constitute the predominant sediment in the nearshore region of the island. On the windward coast, the percentage of biogenous material was so high that the terrigenous fraction was rarely visible to the unaided eye.

Most samples were composed of medium and coarse grain sand containing $80 \%$ to $95 \%$ calcium carbonate of biogenous origin. Analysis by $\mathrm{X}$-ray diffraction showed that, mineralogically, the biogenous material was composed of three components: aragonite, magnesium-rich calcite, and magnesium-poor calcite. Terrigenous sediments were common only on the lee coasts of the island, especially in the vicinity of and down current from river mouths. The terrigenous material consisted mainly of olivine grains and sand-size, partially-weathered feldspathic rock fragments, plus a small percentage of opaque iron oxides.

A brief description of the size distributions of the sands follows, together with their biological, chemical, and mineralogical analyses. Detailed data on each sample are listed in Table 2.

\section{Size Distribution}

The size distributions of all the sediments were determined by the Emery settling tube (Poole et al., 1951), and some, especially the coarsest, were also analyzed by sieving. The median diameter of the particle size distribution is expressed in microns, and measures of the standard deviation (sorting) and skewness are given in the graphic phi notation of Inman (1952). Sieving was necessary in order to obtain the parameters for the coarser samples. Since sieve and settling tube analyses are based on different properties of the sediment, the parameters obtained by the two methods are only roughly comparable. For example, the partly hollow tests of foraminifera are retained in a 
TABLE 2

Location, Type, Size Distribution, AND Composition of SAMPles

\begin{tabular}{|c|c|c|c|c|c|c|c|c|c|c|c|}
\hline \multirow[b]{2}{*}{$\begin{array}{l}\text { SAMPLE } \\
\text { NUMBER }\end{array}$} & \multirow[b]{2}{*}{ LOCATION } & \multirow[b]{2}{*}{$\begin{array}{c}\text { TYPE } \\
\text { SAMPLE }\end{array}$} & \multirow[b]{2}{*}{$\begin{array}{l}\text { BEACH } \\
\text { SLOPE, } \\
\text { TANGENT }\end{array}$} & \multicolumn{3}{|c|}{ SIZE DISTRIBUTIONं } & \multirow[b]{2}{*}{$\begin{array}{c}\% \\
\text { CARBONATE }\end{array}$} & \multicolumn{3}{|c|}{ CARBONATE FRACTION $\S$} & \multirow{2}{*}{$\begin{array}{l}\text { OLIVINE TO } \\
\text { FELDSPAR } \\
\text { PEAK HEIGHT } \\
\text { RATIO } \$\end{array}$} \\
\hline & & & & $\begin{array}{l}\text { Median } \\
\text { Microns }\end{array}$ & $\sigma_{\phi}$ & $a_{\phi}$ & & $\begin{array}{c}\% \\
\text { Aragonite }\end{array}$ & $\begin{array}{c}\% \\
\text { Mg-Rich } \\
\text { Calcite }\end{array}$ & $\begin{array}{c}\% \\
\text { Mg-Poor } \\
\text { Calcite }\end{array}$ & \\
\hline 1 & $\begin{array}{r}\text { Barking } \\
\text { Sands }\end{array}$ & B & .067 & $\begin{array}{c}253 \\
(227) \\
\end{array}$ & $\begin{array}{l}.31 \\
(.310) \\
\end{array}$ & $\begin{array}{l}.0 \\
(.0) \\
\end{array}$ & 72.8 & 41 & 59 & trace & $\sim .5$ \\
\hline 2 & $\begin{array}{r}\text { Barking } \\
\text { Sands }\end{array}$ & B & .101 & $\begin{array}{c}309 \\
(297)\end{array}$ & $\begin{array}{l}.39 \\
(.385)\end{array}$ & $\begin{array}{l}-.08 \\
(-.026)\end{array}$ & 76.0 & & & & \\
\hline 3 & $\begin{array}{r}\text { Barking } \\
\text { Sands }\end{array}$ & $\mathrm{D}$ & & $\begin{array}{c}451 \\
(435) \\
\end{array}$ & $\begin{array}{c}.48 \\
(.465)\end{array}$ & $\begin{array}{l}-.02 \\
(+.043)\end{array}$ & 82.7 & & & & \\
\hline 4 & $\begin{array}{r}\text { Barking } \\
\text { Sands }\end{array}$ & B & .14 & $\begin{array}{c}473 \\
(451) \\
\end{array}$ & $\begin{array}{c}.58 \\
(.50) \\
\end{array}$ & $\begin{array}{l}-.02 \\
(-.18) \\
\end{array}$ & 74.8 & & & & \\
\hline 5 & $\begin{array}{r}\text { Barking } \\
\text { Sands }\end{array}$ & B & & $\begin{array}{c}651 \\
(637) \\
\end{array}$ & $\begin{array}{c}.48 \\
(.65) \\
\end{array}$ & $\begin{array}{l}-.25 \\
(.0)\end{array}$ & 86.8 & & & & \\
\hline 6 & $\begin{array}{r}\text { Barking } \\
\text { Sands }\end{array}$ & $\mathrm{BR}$ & & \multicolumn{2}{|c|}{ beach rock } & & 85.4 & 27 & 73 & trace & \\
\hline 7 & $\begin{array}{l}\text { Nohili } \\
\text { Point }\end{array}$ & $\mathrm{B}$ & .085 & $\begin{array}{c}707 \\
(646) \\
\end{array}$ & $\begin{array}{c}.61 \\
(.48) \\
\end{array}$ & $\begin{array}{c}+.02 \\
(-.01)\end{array}$ & 85.0 & & . & & \\
\hline 8 & Mana & B & & $\begin{array}{c}779 \\
(747) \\
\end{array}$ & $\begin{array}{l}.45 \\
(.42) \\
\end{array}$ & $\begin{array}{c}+.02 \\
(-.01) \\
\end{array}$ & 89.6 & 28.7 & 62.8 & 8.5 & \\
\hline 9 & Mana & B & & $\begin{array}{c}245 \\
(243) \\
\end{array}$ & $\begin{array}{l}.56 \\
(.72) \\
\end{array}$ & $\begin{array}{l}-.34 \\
(-.42)\end{array}$ & 88.8 & & & & \\
\hline 10 & Mana & B & & $\begin{array}{c}243 \\
(230) \\
\end{array}$ & $\begin{array}{l}.38 \\
(.46) \\
\end{array}$ & $\begin{array}{c}-.24 \\
(-.21) \\
\end{array}$ & 87.3 & & & & \\
\hline 11 & Kokole & B & .13 & $\begin{array}{c}281 \\
(330) \\
\end{array}$ & $\begin{array}{l}.51 \\
(.51) \\
\end{array}$ & $\begin{array}{l}-.27 \\
(-.20)\end{array}$ & 88.1 & & & & \\
\hline 12 & Kekaha & B & .101 & $\begin{array}{c}257 \\
(262) \\
\end{array}$ & $\begin{array}{l}.56 \\
(.64) \\
\end{array}$ & $\begin{array}{l}-.26 \\
(-.12) \\
\end{array}$ & 86.3 & & & & \\
\hline 13 & Kekaha & B & .096 & $\begin{array}{c}262 \\
(257) \\
\end{array}$ & $\begin{array}{l}.51 \\
(.55) \\
\end{array}$ & $\begin{array}{l}-.31 \\
(-.08)\end{array}$ & 86.1 & 39 & 61 & trace & $\sim .3$ \\
\hline 14 & Kekaha & B & .13 & $\begin{array}{l}297 \\
(337)\end{array}$ & $\begin{array}{c}.48 \\
(.49)\end{array}$ & $\begin{array}{l}-.23 \\
(0.00)\end{array}$ & 76.9 & & & & \\
\hline
\end{tabular}


TABLE 2 (continued)

Location, Type, Size Distribution, AND Composition of SAMPLES

\begin{tabular}{|c|c|c|c|c|c|c|c|c|c|c|c|}
\hline \multirow[b]{2}{*}{$\begin{array}{l}\text { SAMPLE } \\
\text { NUMBER }\end{array}$} & \multirow[b]{2}{*}{ LOCATION } & \multirow[b]{2}{*}{$\begin{array}{c}\text { TYPE } \\
\text { SAMPLE }\end{array}$} & \multirow[b]{2}{*}{$\begin{array}{l}\text { BEACH } \\
\text { SLOPE, } \\
\text { TANGENT }\end{array}$} & \multicolumn{3}{|c|}{ SIZE DISTRIBUTION $\dagger$} & \multirow[b]{2}{*}{$\begin{array}{c}\% \\
\text { CARBONATE } *\end{array}$} & \multicolumn{3}{|c|}{ CARBONATE FRACTION $\S$} & \multirow{2}{*}{$\begin{array}{c}\text { OLIVINE TO } \\
\text { FELDSPAR } \\
\text { PEAK HEIGHT } \\
\text { RATIO }\end{array}$} \\
\hline & & & & $\begin{array}{l}\text { Median } \\
\text { Microns }\end{array}$ & $\sigma_{\phi}$ & $a_{\phi}$ & & $\begin{array}{c}\% \\
\text { Aragonite }\end{array}$ & $\begin{array}{c}\% \\
\text { Mg-Rich } \\
\text { Calcite }\end{array}$ & $\begin{array}{c}\% \\
\text { Mg-Poor } \\
\text { Calcite }\end{array}$ & \\
\hline 15 & Kekaha & B & & $\begin{array}{c}429 \\
(412) \\
\end{array}$ & $\begin{array}{c}.46 \\
(.42) \\
\end{array}$ & $\begin{array}{l}-.13 \\
(+.10)\end{array}$ & 52.9 & 29 & 71 & & $<.05$ \\
\hline 16 & Kekaha & B1 & .19 & $\begin{array}{c}574 \\
(555) \\
\end{array}$ & $\begin{array}{c}.69 \\
(.40) \\
\end{array}$ & $\begin{array}{l}-.05 \\
(+.05)\end{array}$ & 34.9 & & & & \\
\hline 17 & Oomano & B & & $\begin{array}{c}398 \\
(401)\end{array}$ & $\begin{array}{c}.49 \\
(.30)\end{array}$ & $\begin{array}{l}-.29 \\
(-.25)\end{array}$ & 32.0 & & & & $<.05$ \\
\hline 18 & Oomano & $\mathrm{BR}$ & & \multicolumn{2}{|c|}{ beach rock } & & 44.8 & 17 & 83 & & $<.07$ \\
\hline 19 & Waimea & B & .13 & $\begin{array}{c}382 \\
(409) \\
\end{array}$ & $\begin{array}{c}.68 \\
(.44) \\
\end{array}$ & $\begin{array}{l}-.36 \\
(-.70)\end{array}$ & 18.8 & & & & \\
\hline 20 & Waimea & B & & $\begin{array}{c}240 \\
(200) \\
\end{array}$ & $\begin{array}{l}.43 \\
(.47) \\
\end{array}$ & $\begin{array}{l}-.21 \\
(-.12)\end{array}$ & 7.7 & & & & \\
\hline 21 & Waimea & B & .11 & $\begin{array}{c}277 \\
(276) \\
\end{array}$ & $\begin{array}{l}.53 \\
(.57)\end{array}$ & $\begin{array}{c}+.05 \\
(+.08)\end{array}$ & 14.7 & & & & \\
\hline 22 & Waimea & B & .13 & $\begin{array}{c}335 \\
(274) \\
\end{array}$ & $\begin{array}{c}.69 \\
(.67) \\
\end{array}$ & $\begin{array}{l}-.13 \\
(0.00)\end{array}$ & 4.4 & & & & \\
\hline 23 & Waimea & B & .12 & $\begin{array}{c}304 \\
(344) \\
\end{array}$ & $\begin{array}{c}.62 \\
(.59) \\
\end{array}$ & $\begin{array}{l}-.25 \\
(+.14)\end{array}$ & 6.9 & & & & \\
\hline 24 & Waimea & B & .13 & $\begin{array}{c}390 \\
(319)\end{array}$ & $\begin{array}{c}.81 \\
(.65) \\
\end{array}$ & $\begin{array}{l}-.21 \\
(-.05)\end{array}$ & 1.3 & & & & .69 \\
\hline 25 & Waimea & B & .16 & $\begin{array}{c}293 \\
(304) \\
\end{array}$ & $\begin{array}{l}.50 \\
(.50) \\
\end{array}$ & $\begin{array}{l}-.10 \\
(-.06)\end{array}$ & 00.5 & & & & \\
\hline 26 & Waimea & $\mathrm{S}$ & & $\begin{array}{c}470 \\
(335)\end{array}$ & $\begin{array}{c}.63 \\
(.55) \\
\end{array}$ & $\begin{array}{l}-.16 \\
(-.02)\end{array}$ & 0.7 & & & & \\
\hline 27 & $\begin{array}{c}\text { Makaweli } \\
\text { Landing }\end{array}$ & $\mathrm{B} 1$ & .17 & 330 & .38 & -.03 & 84.6 & 42 & 58 & trace & \\
\hline 28 & Koki & $\mathrm{B}$ & .17 & 824 & .54 & -.02 & 86.0 & & & & \\
\hline 29 & $\begin{array}{l}\text { Kuunakaiole } \\
\text { Park }\end{array}$ & $\mathrm{B} 1$ & .17 & 532 & .35 & -.11 & 90.8 & & & & \\
\hline
\end{tabular}


TABLE 2 (continued)

Location, Type, Size Distribution, ANd Composition of SAmples

\begin{tabular}{|c|c|c|c|c|c|c|c|c|c|c|c|}
\hline \multirow[b]{2}{*}{$\begin{array}{l}\text { SAMPLE } \\
\text { NUMBER } \\
\end{array}$} & \multirow[b]{2}{*}{ LOCATION } & \multirow[b]{2}{*}{$\begin{array}{c}\text { TYPE } \\
\text { SAMPLE* } \\
\end{array}$} & \multirow[b]{2}{*}{$\begin{array}{l}\text { BEACH } \\
\text { SLOPE, } \\
\text { TANGENT }\end{array}$} & \multicolumn{3}{|c|}{ SIZE DISTRIBUTION $\dagger$} & \multirow[b]{2}{*}{$\begin{array}{c}\% \\
\text { CARBONATE } \ddagger \\
\end{array}$} & \multicolumn{3}{|c|}{ CARBONATE FRACTION $\$$} & \multirow{2}{*}{$\begin{array}{c}\text { OLIVINE TO } \\
\text { FELDSPAR } \\
\text { PEAK HEIGHT } \\
\text { RATIO } \$ \\
\end{array}$} \\
\hline & & & & $\begin{array}{l}\text { Median } \\
\text { Microns } \\
\end{array}$ & $\sigma_{\phi}$ & $a_{\phi}$ & & $\begin{array}{c}\% \\
\text { Aragonite }\end{array}$ & $\begin{array}{c}\% \\
\text { Mg-Rich } \\
\text { Calcite }\end{array}$ & $\begin{array}{c}\% \\
\text { Mg-Poor } \\
\text { Calcite }\end{array}$ & \\
\hline 30 & $\begin{array}{c}\text { Hanapepe } \\
\text { Bay }\end{array}$ & B2 & .17 & 259 & .46 & +.04 & 4.2 & & & & \\
\hline 31 & Wahiwa Bay & B & .17 & 321 & .48 & -.17 & 52.7 & 28 & 52 & 20.2 & $<.15$ \\
\hline 32 & Nahunakueu & $\mathrm{B}$ & .13 & 289 & .29 & -.17 & 79.7 & & & & \\
\hline 33 & Lawai Bay & $\mathrm{B}$ & .072 & 182 & .32 & +.06 & 80.8 & & & & \\
\hline 34. & Poipu & $\mathrm{B} 2$ & .17 & 277 & .58 & -.33 & 88.6 & & & & \\
\hline 35 & Makahuena & $\mathrm{BR}$ & & \multicolumn{2}{|c|}{ beach rock } & & 91.5 & 25.8 & 69.7 & 4.5 & \\
\hline 36 & Makahuena & B & .11 & 285 & .34 & -.09 & 89.1 & & & & \\
\hline 37 & Kapunakea & B & .17 & 295 & .32 & -.22 & 89.0 & & & & \\
\hline 38 & Kipu Kai & B & .11 & 222 & .30 & -.20 & 89.1 & & & & \\
\hline 39 & Kipu Kai & B2 & .16 & 196 & .28 & -.19 & 86.7 & & & & \\
\hline 40 & Kipu Kai & DR & & \multicolumn{2}{|c|}{ dune rock } & & 88.9 & trace & 0.0 & 100 & \\
\hline 41 & Kipu Kai & $\mathrm{BR}$ & & \multicolumn{2}{|c|}{ beach rock } & & 90.8 & 45 & 45 & 10 & \\
\hline 42 & Kipu Kai & B & .14 & $\begin{array}{c}518 \\
(543) \\
\end{array}$ & $\begin{array}{c}.90 \\
(.69) \\
\end{array}$ & $\begin{array}{l}-.06 \\
(+.07)\end{array}$ & 87.8 & & & & \\
\hline 43 & Kipu Kai & B & & $(1240)$ & $(.35)$ & $(-.06)$ & 91.2 & 25 & 75 & & \\
\hline 44 & Nawiliwili & $B$ & .096 & 162 & .63 & -.13 & 87.0 & & & . & \\
\hline 45 & Hanamaulu & $\mathrm{B}$ & .085 & 139 & .47 & -.06 & 78.2 & 33.3 & 54.4 & 12.2 & \\
\hline 46 & $\begin{array}{l}\text { Military } \\
\quad \text { Reservation } \\
\end{array}$ & B1 & .19 & 387 & .48 & -.21 & 90.0 & & & & \\
\hline 47 & Wailua & $\mathrm{B}$ & .15 & 342 & .40 & -.13 & 80.0 & & & & \\
\hline 48 & Kapaa & B1 & .16 & 361 & .46 & -.19 & 91.1 & 29.5 & 50.1 & 20.4 & \\
\hline 49 & Kapaa & $\mathrm{B} 1$ & .18 & 500 & .39 & 0.0 & 93.3 & 36.8 & 46.7 & 16.5 & \\
\hline 50 & Kapaa & $\mathrm{O}$ & & 371 & .40 & -.02 & 90.0 & 33.3 & 48.0 & 18.7 & \\
\hline 51 & Kapaa & $\mathrm{B} 1$ & .16 & 420 & .57 & -15 & 92.4 & 30.0 & 53.2 & 16.8 & \\
\hline
\end{tabular}


TABLE 2 (continued)

LOCATION, Type, Size Distribution, AND COMPosîtTION OF SAMPles

\begin{tabular}{|c|c|c|c|c|c|c|c|c|c|c|c|}
\hline \multirow[b]{2}{*}{$\begin{array}{c}\text { SAMPLE } \\
\text { NUMBER }\end{array}$} & \multirow[b]{2}{*}{ LOCATION } & \multirow[b]{2}{*}{$\begin{array}{c}\text { TYPE } \\
\text { SAMPLE* }\end{array}$} & \multirow[b]{2}{*}{$\begin{array}{l}\text { BEACH } \\
\text { SLOPE, } \\
\text { TANGENT }\end{array}$} & \multicolumn{3}{|c|}{ SIZE DISTRIBUTIONं } & \multirow[b]{2}{*}{$\begin{array}{c}\% \\
\text { CARBONATE }\end{array}$} & \multicolumn{3}{|c|}{ CARBONATE FRACTION $\S$} & \multirow{2}{*}{$\begin{array}{l}\text { OLIVINE TO } \\
\text { FELDSPAR } \\
\text { PEAK HEIGHT } \\
\text { RATIO } \$\end{array}$} \\
\hline & & & & $\begin{array}{l}\text { Median } \\
\text { Microns }\end{array}$ & $\sigma_{\phi}$ & $a_{\phi}$ & & $\begin{array}{c}\% \\
\text { Aragonite }\end{array}$ & $\begin{array}{c}\% \\
\text { Mg-Rich } \\
\text { Calcite }\end{array}$ & $\begin{array}{c}\% \\
\text { Mg-Poor } \\
\text { Calcite }\end{array}$ & \\
\hline 52 & Kapaa & $\mathrm{B} 1$ & .16 & 500 & .53 & -.10 & 93.3 & 29.3 & 52.1 & 18.6 & \\
\hline 53 & Kapaa & $\mathrm{B} 1$ & .19 & 490 & .60 & -.06 & 89.7 & 25.8 & 57.5 & 16.7 & \\
\hline 54 & Kapaa & B2 & .19 & 525 & .55 & 0.0 & 93.8 & 27.0 & 54.9 & 18.0 & \\
\hline 55 & Kapaa & B1 & .17 & $\begin{array}{c}555 \\
(651)\end{array}$ & $\begin{array}{l}.52 \\
(.33) \\
\end{array}$ & $\begin{array}{l}-.06 \\
(+.09)\end{array}$ & 92.7 & 26.6 & 56.5 & 16.9 & \\
\hline 56 & Kapaa & RF & & $\begin{array}{c}785 \\
(901)\end{array}$ & $\begin{array}{c}.45 \\
(.37)\end{array}$ & $\begin{array}{l}-.33 \\
(-.06)\end{array}$ & 92.7 & 23.1 & 53.8 & 23.1 & \\
\hline 57 & Kapaa & $\mathrm{B} 1$ & .16 & 595 & .61 & -.05 & 91.3 & 26.7 & 57.2 & 16.1 & \\
\hline 58 & Kapaa & RF & & $(599)$ & $(.51)$ & $(-.37)$ & 87.9 & 27.7 & 53.8 & 18.5 & \\
\hline 59 & Kapaa & $\mathrm{O}$ & & $(829)$ & $(.37)$ & $(-.19)$ & 90.7 & 22.2 & 54.5 & 23.2 & \\
\hline 60 & Kapaa & $\mathrm{O}$ & & $\begin{array}{c}785 \\
(801)\end{array}$ & $\begin{array}{c}.62 \\
(.44)\end{array}$ & $\begin{array}{l}-.11 \\
(-.14)\end{array}$ & 95.0 & 25.6 & 54.3 & 20.1 & \\
\hline 61 & Kapaa & B1 & .19 & 493 & .60 & 0.0 & 93.3 & 29.8 & 55.4 & 14.8 & \\
\hline 62 & Kapaa & $\mathrm{B} 1$ & .17 & $\begin{array}{c}669 \\
(807) \\
\end{array}$ & $\begin{array}{c}.60 \\
(.38) \\
\end{array}$ & $\begin{array}{c}+.23 \\
(+.18)\end{array}$ & 90.3 & 27.2 & 60.4 & 12.4 & \\
\hline 63 & Kapaa & RF & & $(959)$ & $(.53)$ & $(-.11)$ & 88.2 & 26.7 & 62.3 & 11.0 & \\
\hline 64 & Kealia & $\mathrm{B}$ & .17 & 289 & .27 & -.33 & 88.5 & & & & \\
\hline 65 & Anahola Bay & $\mathrm{B}$ & .17 & 335 & .46 & -.30 & 89.7 & & & & \\
\hline 66 & Moloaa Bay & $\mathrm{B}$ & .12 & 603 & .60 & -.13 & 88.9 & & & & \\
\hline 67 & Kilauea Bay & B & & 254 & .42 & -.07 & 75.9 & & & & \\
\hline 68 & $\begin{array}{l}\text { Kalihiwai } \\
\text { Bay }\end{array}$ & B & .14 & 242 & .36 & -.33 & 71.8 & & & & \\
\hline 69 & Kalihikai & $\mathrm{B} 1$ & .13 & 149 & .34 & -.09 & 88.0 & 19 & 81 & trace & \\
\hline 70 & Anini & $\mathrm{B}$ & & 369 & .97 & -.19 & 85.4 & 46 & 54 & 00.0 & \\
\hline 71 & Hanalei Bay & $\mathrm{B}$ & .11 & 172 & .34 & -.29 & 62.0 & 52 & 48 & trace & $<.2$ \\
\hline 72 & Hanalei Bay & B & .037 & 190 & .39 & -.13 & 48.9 & & & -2 & \\
\hline
\end{tabular}


TABLE 2 (continued)

LOCATION, TyPe, Size Distribution, AND COMPOSITION OF SAMPLES

\begin{tabular}{|c|c|c|c|c|c|c|c|c|c|c|c|}
\hline \multirow[b]{2}{*}{$\begin{array}{c}\text { SAMPLE } \\
\text { NUMBER }\end{array}$} & \multirow[b]{2}{*}{ LOCATION } & \multirow[b]{2}{*}{$\begin{array}{c}\text { TYPE } \\
\text { SAMPLE* }\end{array}$} & \multirow[b]{2}{*}{$\begin{array}{l}\text { BEACH } \\
\text { SLOPE, } \\
\text { TANGENT }\end{array}$} & \multicolumn{3}{|c|}{ SIZE DISTRIBUTION† } & \multirow[b]{2}{*}{$\begin{array}{c}\% \\
\text { CARBONATE }\end{array}$} & \multicolumn{3}{|c|}{ CARBONITE FRACTION $\S$} & \multirow{2}{*}{$\begin{array}{l}\text { OLIVINE TO } \\
\text { FELDSPAR } \\
\text { PEAK HEIGHT } \\
\text { RATIO } \$\end{array}$} \\
\hline & & & & $\begin{array}{l}\text { Median } \\
\text { Microns }\end{array}$ & $\sigma_{\phi}$ & $a_{\phi}$ & & $\begin{array}{c}\% \\
\text { Aragonite }\end{array}$ & $\begin{array}{c}\% \\
\text { Mg-Rich } \\
\text { Calcite }\end{array}$ & $\begin{array}{c}\% \\
\text { Mg-Poor } \\
\text { Calcite }\end{array}$ & \\
\hline 73 & Hanalei Bay & $\mathrm{B}$ & .101 & 341 & .51 & -.20 & 74.2 & & & & $<.2$ \\
\hline 74 & Hanalei Bay & B & & 330 & .55 & -.04 & 76.2 & & & & \\
\hline 75 & Hanalei Bay & $\mathrm{B}$ & .13 & 364 & .54 & -.11 & 67.4 & & & & \\
\hline 76 & Lumahai Bay & $\mathrm{B}$ & .18 & 611 & .71 & -.14 & 62.5 & 29 & 71 & trace & .2 \\
\hline 77 & Lumahai Bay & $\mathrm{B}, \mathrm{BC}$ & & 475 & .56 & -.18 & 10.7 & & & & 3.9 \\
\hline 78 & Wainiha Bay & $\mathrm{B}$ & .16 & $(1347)$ & $(.27)$ & $(-.07)$ & 54.6 & 25 & 75 & 0 & $<.06$ \\
\hline 79 & Wainiha Bay & $\mathrm{B}, \mathrm{BC}$ & & 369 & .55 & -.22 & 11.7 & & & & 2.7 \\
\hline 80 & Wainiha Bay & $\mathrm{B}$ & .19 & 717 & .43 & +.14 & 92.5 & & & & \\
\hline 81 & Haena Point & B1 & .19 & 871 & .39 & +.32 & 90.7 & & & & \\
\hline 82 & Haena & B & .16 & $\begin{array}{l}819 \\
(707)\end{array}$ & $(.64)$ & $(-.09)$ & 89.1 & & & & \\
\hline 83 & Ke'e & B1 & .20 & $\begin{array}{c}369 \\
(309) \\
\end{array}$ & $\begin{array}{l}.38 \\
(.35) \\
\end{array}$ & $\begin{array}{l}-.18 \\
(0.00)\end{array}$ & 6.8 & & & & 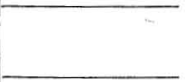 \\
\hline $84 c$ & Ke'e & RF & & 344 & .62 & -.40 & 64.4 & & & & \\
\hline $84 t$ & Ke'e & RF & & 295 & .66 & -.50 & 53.0 & & & & \\
\hline 85 & Hanakapiai & B & & 620 & .78 & +.09 & 85.3 & & & & \\
\hline 86 & Hanakapiai & B & .043 & 242 & .33 & -.12 & 23.8 & & & & \\
\hline 87 & Hanakapiai & $\mathrm{S}$ & & $(3732)$ & & & 0.5 & & & & \\
\hline
\end{tabular}

* Sample type: B beach sand; B1, beach sand (protected by fringing reef); B2, beach sand (very protected bay); BC, heavy mineral concentrate not typical of whole beach; $B R$, beach rock; $D$, dune sand; $D R$, dune rock; $O$, offshore sand; $S$, stream sand; $R F$, reef sand.

$\dagger$ Size parameters enclosed in parentheses were obtained by sieving; unenclosed values were from analysis in an Emery settling tube. Deviation (sorting) and skewness are in raphic phi measures (Inman, 1952).

$\ddagger$ Gasometric analysis (Bien, 1952).

$\S \mathrm{X}$-ray diffraction analysis. 


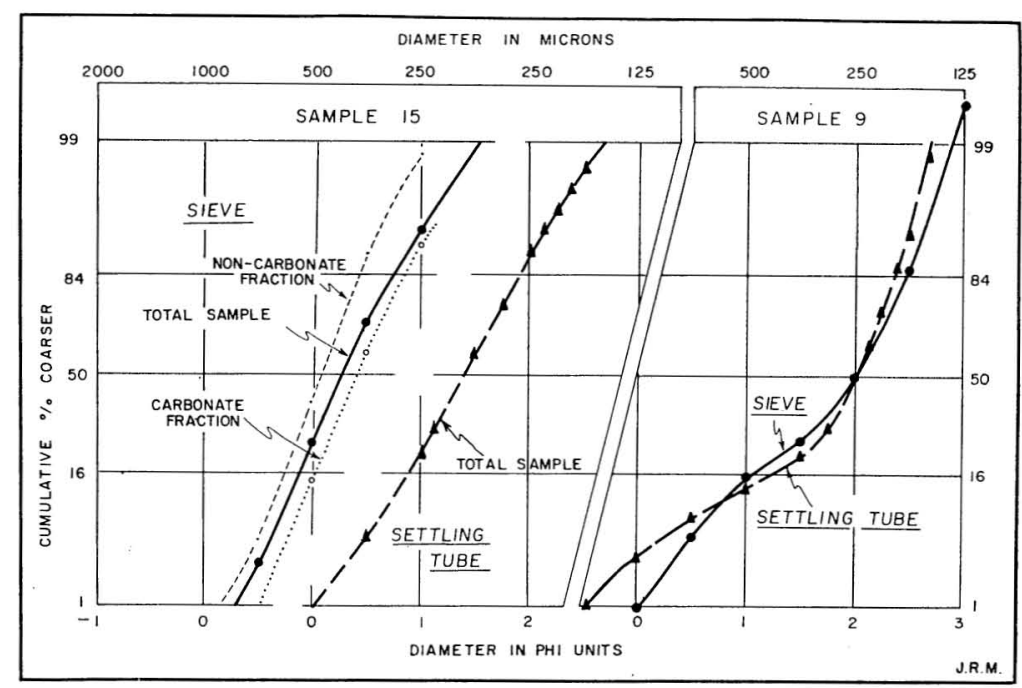

FIG. 5. Size distribution curves for sand samples 9 and 15, showing comparison between settling tube and sieve analysis. Grain size distribution curves are also presented for the carbonate and noncarbonate fractions, which make up $53 \%$ and $47 \%$, respectively, of sample 15 . The size distribution curves for sample 9 ( $89 \%$ calcium carbonate) show a marked bimodal distribution, which is not typical of most highly calcareous samples of Kauai. In this case, the bimodal distribution reflects the differences between the coarse carbonate grains arriving from the north and the finer, but more abundant, carbonate grains from the east.

coarser sieve fraction than their settling velocity would indicate. Comparisons between sieve and settling tube analyses are shown in Figure 5.

The most common beach sediment had a median diameter of about 350 microns, while the median size of all samples ranged from 140 to 1400 microns. Although the size distributions of the samples from the windward and leeward coasts as a group were not markedly different, smaller coastal segments did show significant longshore trends of increasing or decreasing grain size. There is a tendency towards bimodality in sediment size distribution, especially where mixing of sediment from different transport directions occurs southeast of Nohili Point (Sample 9, Fig. 5). Also, the beach face sediments from Kauai, which had an average phi deviation measure of about 0.5, are less well sorted on the average than those from typically continental beaches. Well-sorted sand of similar size from continental beaches would have a phi deviation measure of about 0.3 (Inman, 1949, 1953 ). The more frequent interruption of littoral drift by points and headlands, and the con- tinuous addition of a heterogeneous assortment of material from reefs and from offshore, probably account for this difference in sorting.

The slope of the beach face increases with increasing sand size and with decreasing wave height and intensity. Beaches like those at $\mathrm{Ka}$ paa, which are protected by broad fringing reefs from the direct attack of large breakers, were consistently steeper than more exposed beaches. Also, beaches inside protected bays (as Station 30, Hanapepe; Table 2) were steeper than those exposed to the open sea (as Stations 1-7 at Nohili, and Station 86 at Hanakapiai). The relation between beach slope and sand size for various type beaches is given in Figure 6.

\section{Biological Analysis}

The only completely identifiable biogenous components present in significant quantities in the sands were the benthic foraminifera Heterostegina suborbicularis and Amphistegina madagascariensis. Other positively recognizable components, including other foraminifera, echinoid spines, sponge spicules, gastropod shells and 


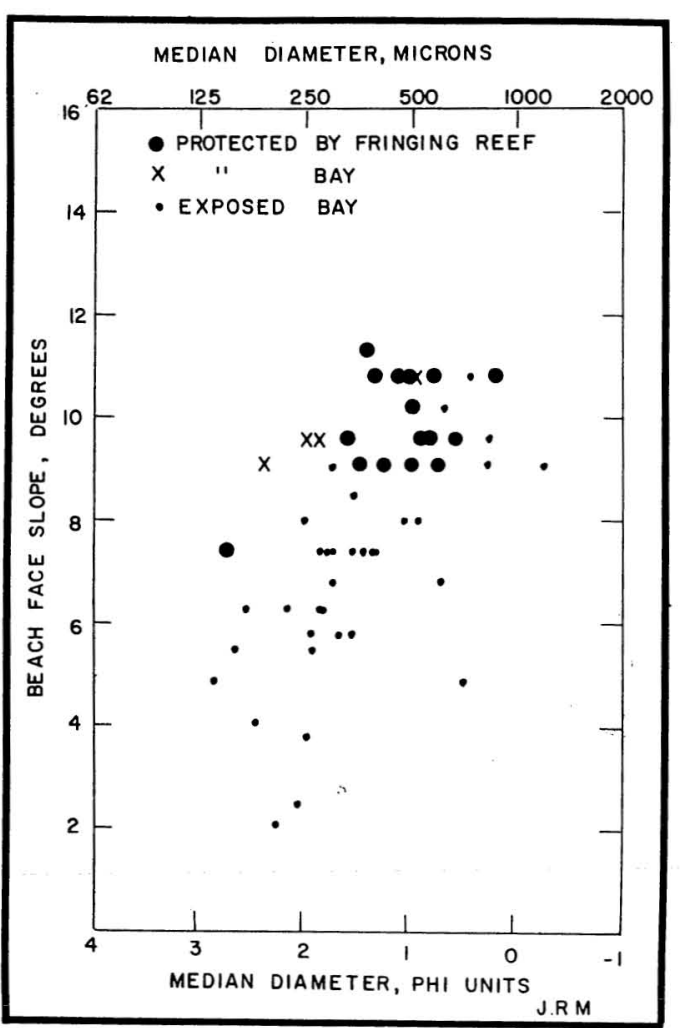

FIG. 6. Relation between beach slope and sand size. Exposed beaches generally have lower slopes than protected beaches.

opercula, pelecypod valves, and bryozoan fragments, made up less than $5 \%$ of the samples. Most of the remaining nonterrigenous component of the sand consisted of rounded calcareous grains, sometimes etched, pitted, or stained.

A rough count indicated that Amphistegina and Heterostegina accounted for up to $20 \%$ of the grains in some of the samples. Since these foraminifera occurred most commonly in the size range of 500 to 1500 microns, they were more abundant in the coarser sediments. $\mathrm{Am}$ phistegina were most common in the finer half of this range.

Several indentifiable skeletal or shell fragments, collected from the reefs and beaches of Kauai, or in one case from $\mathrm{Oahu}$, were examined by X-ray diffraction. The results and a review of the literature suggest that, in this area, the aragonite is secreted principally by corals and molluscs; the magnesium-rich calcite by foraminifera, coralline algae, and echinoids; and the magnesium-poor calcite by molluscs and by $\mathrm{Am}$ phistegina madagascariensis.

\section{Chemical and Mineralogical Analysis}

All of the sand samples were examined qualitatively, and representative samples were also examined quantitatively to determine the chemical, mineralogical, and biological composition. The carbonate content of all of the samples was determined to an accuracy of about $1 \%$, using a gasometric technique described by Bien (1952). Mineralogical determinations were made with a petrographic microscope and by X-ray diffraction, using a North American Phillips Diffractometer.

TOTAL CARBONATE: The total carbonate content varied from $0.5 \%$, in the river bottom samples, to $95 \%$ in some beach samples. Fifty-two of the samples had carbonate contents greater than $80 \%$, while only 17 contained less than $50 \% \mathrm{CaCO}_{3}$. Although high carbonate values occurred on all coasts, samples from the east coast were composed almost entirely of biogenous material. The sands showing the highest carbonate values on the north, south, and Mana coasts were taken from those beaches which were most distant from large streams (Table 2). On the east (windward) coast and on the eastern part of the north coast, the presence of large streams appeared to have little effect on the carbonate content of the beach sediments. Low carbonate values were typical of the beaches west of the Waimea River mouth on the south coast between sample stations 15 and 25 and, to a lesser degree, in Hanalei Bay on the north coast.

CALCITE: The X-ray patterns of the sand samples showed that the calcite frequently consisted of two components, magnesium-rich and magnesium-poor calcite (Fig. 7). A quantitative determination of the amount of magnesium carbonate in each of the calcite components (i.e., the mineral species of the component) was made by comparing the peak location of each component with the curve published by Goldsmith et al. (1955: fig. 1). In the Kauai samples, the magnesium-poor peak represents a calcite containing from $2 \%$ to $3 \% \mathrm{MgCO}_{3}$; the magnesium-rich peak represents a calcite containing 
about $13 \% \mathrm{MgCO}_{3}$. Determination of the amount of both of the calcite components, and of the aragonite component in the carbonate fraction of each sample, was made by comparing the peak ratios of these components prepared from known mixtures of the components (Gayman, in preparation; Lowenstam, 1954). Although recognized corrections have not been made, it is thought that the relative quantitative results are significant.

In all of the samples examined, with the exception of one dune rock sample (40), the magnesium-rich calcite was much more abundant than the magnesium-poor calcite. This rock was obtained from a lithified relic dune which now forms a headland (sample station 40) of Kipu Kai Valley, south of Lihue. With this one exception, magnesium-poor calcite never constituted more than $25 \%$ of the carbonate content of any sample.

Magnesium-rich calcite was always present in the unconsolidated samples, and it was fre-

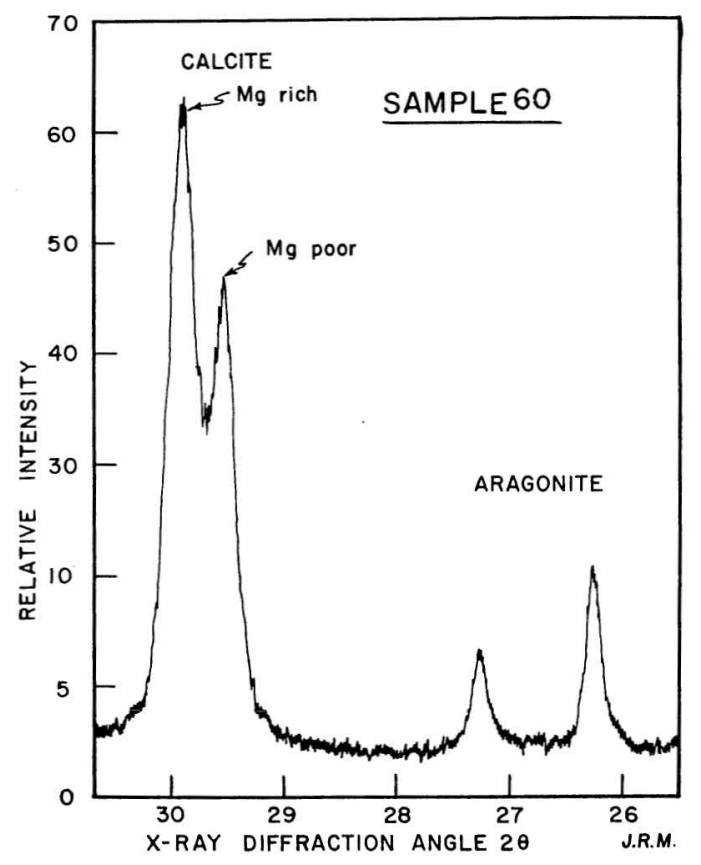

FIG. 7. X-ray diffraction analysis of sample 60, showing the magnesium-rich and magnesium-poor peaks and the two aragonite peaks. The sample was taken in a water depth of $27 \mathrm{ft}$ outside of Kapaa Reef (Fig. 8). quently the only calcite component found. Magnesium-poor calcite was absent from the north and Napali coasts, and was abundant only in the dune rock and on the east coast in the vicinity of Kapaa Reef. The distribution of magnesiumpoor calcite in the Kapaa area and the anomalous abundance of this component in the dune rock (sample 40) are discussed subsequently.

According to Chave (1954a), water temperature and phylogenetic level are principle factors in determining the magnesium content of biogenous calcite. High magnesium content is favored by warmer water and lower phylogenetic level. Temperature has less effect on the magnesium content of the higher phylogenetic levels. Blackman and Todd (1959) show magnesium content in foraminifera to vary with family. Heterostegina, one of the two common species of foraminifera in the samples, and two unidentified species of coralline algae taken from $\mathrm{Ka}$ paa Reef, were X-rayed and found to contain only magnesium-rich calcite. Conversely, Amphistegina, the other common foraminifera, and a limpet were found to be composed mostly or entirely of magnesium-poor calcite.

ARAgonite: Aragonite is generally considered to be indicative of warm water deposition. It is deposited by three quantitatively significant groups (Lowenstam, 1954): calcareous algae, scleractinian corals, and molluscs. Samples from Kapaa Reef of two different species of coral (Acropora and a scleractinian coral) and one specimen of gastropod (Conus) were X-rayed and found to be pure aragonite. One limpet, mentioned previously, was found to be predominantly magnesium-poor calcite and only $28 \%$ aragonite. Although the aragonite in the carbonate fraction ranged from $19 \%$ to $45 \%$, no systematic variation with depth or direction was apparent.

TERRIGENOUS: A cursory inspection of the differences in the terrigenous mineralogy was made by microscopic and X-ray diffraction techniques. A limited amount of $\mathrm{X}$-ray data on the terrigenous components of the samples is presented in Table 2 in the form of the olivine to feldspar peak height ratios. These data should be regarded as only semiquantitative because of the variation in peak height introduced by preferred orientation of the feldspar crystals. 
Presumably, a difference in the degree of chemical weathering accounts for the differences in mineralogy between the terrigenous sediments on the windward and leeward coasts. Plagioclaserich sands occur near the Waimea River mouth, while on the north coast the same size grades of sand are plagioclase-poor and olivine-rich; however, there appears to be little difference in the average parent rock in the two source areas. It seems likely that a more rapid decomposition of feldspar, relative to olivine, takes place because the feldspar occurs in smaller mineral grains. The plagioclase crystals, found only in sand-size rock fragments, are several orders of magnitude smaller than the olivine grains found in the beach sands. The plagioclase crystals are abundant in medium- and fine-grain beach sands only in the Waimea area. Apparently the easily decomposable volcanic rock fragments in these size ranges are brought to the shore in mass only where chemical processes are at a minimum. On Kauai a maximum in sediment yield and a minimum in chemical weathering occur only in the arid Waimea drainage basin.

\section{Beach and Dune Rock}

The distribution of beach rock on Kauai is described by Emery and Cox (1956) in a comprehensive study of beach rock in the Hawaiian Islands. They examined 72 miles of sandy beaches on Kauai and reported beach rock along 21 miles, or about $13 \%$ of the entire coastline. The total occurrence of beach rock may be even greater, as the presence of beach rock is frequently obscured by a covering of loose clastic sand. During the present study, a large outcrop of beach rock that was surveyed and sampled (No. 18) at Oomana Point in 1955, was completely covered with fresh sand when the site was revisited in 1959. Also, in the vicinity of samples 38 and 39, it was found that a $1 / 4$-mile section of beach rock was covered with a uniform layer of fresh sand, about 4 inches thick. Here the slope and general attitude of the beach rock was the same as that of the fresh beach face. Water seeping through the beach face was tasted and found to be fresh, indicating that its origin was the fresh water ponded behind the beach ridge.
In fact, there is some correlation on the is. land of Kauai between beach rock outcrops and areas of present or past ponding of fresh water behind calcareous beach ridges. Although it is recognized (Emery and Cox, 1956) that there may be several methods of formation of beach rock, it appears that an important cementation process on Kauai is associated with the evaporation of fresh calcium carbonate-rich waters seeping through the beach face. This occurs most generally when fresh water is ponded in calcareous sediments by sand dunes or beach ridges. Presumably, the fresh or brackish water flowing through the porous calcareous sediments becomes saturated with $\mathrm{CaCO}_{3}$. Evaporation of this water on or near the beach face causes cementation, and rock is formed if the beach is sufficiently stable. This belief is strengthened by the presence of cemented dune sands, which are well above sea level and appear to have been formed in the same manner as the beach rock, with which these cemented sands are commonly associated.

Dune rock (eolianite) was found on the southwest coast of the island, northeast of Nohili Point. Near Nohili the dune rock was poorly cemented and appeared to be contemporaneous with the formation of the present dunes at Barking Sands. In the vicinity of Kipu Kai (sample 40) several rocky points, such as Alligator Head, are composed of well-cemented dune rock, which is now exposed to wave attack. The Kipu Kai dune rock is older than the present shoreline.

Thin section studies of beach and dune rock samples $(6,18,35,40,41)$ confirm the lithologic and biologic similarity of the rock samples and the recent beach sand. The general structure, texture, and composition of the beach rocks suggest that they were formed either very recently, or under the same environmental conditions as those which are now dominant on Kauai beaches. Examination of thin sections and X-ray analysis suggest that the cement is calcitic, in agreement with the findings of Emery and Cox (1956).

The only dune rock sample, 40 , differed from the beach and beach rock samples in that the carbonate fraction is composed entirely of magnesium-poor calcite and the cement was considerably more abundant. It does not seem likely that the single carbonate mineral species now found 


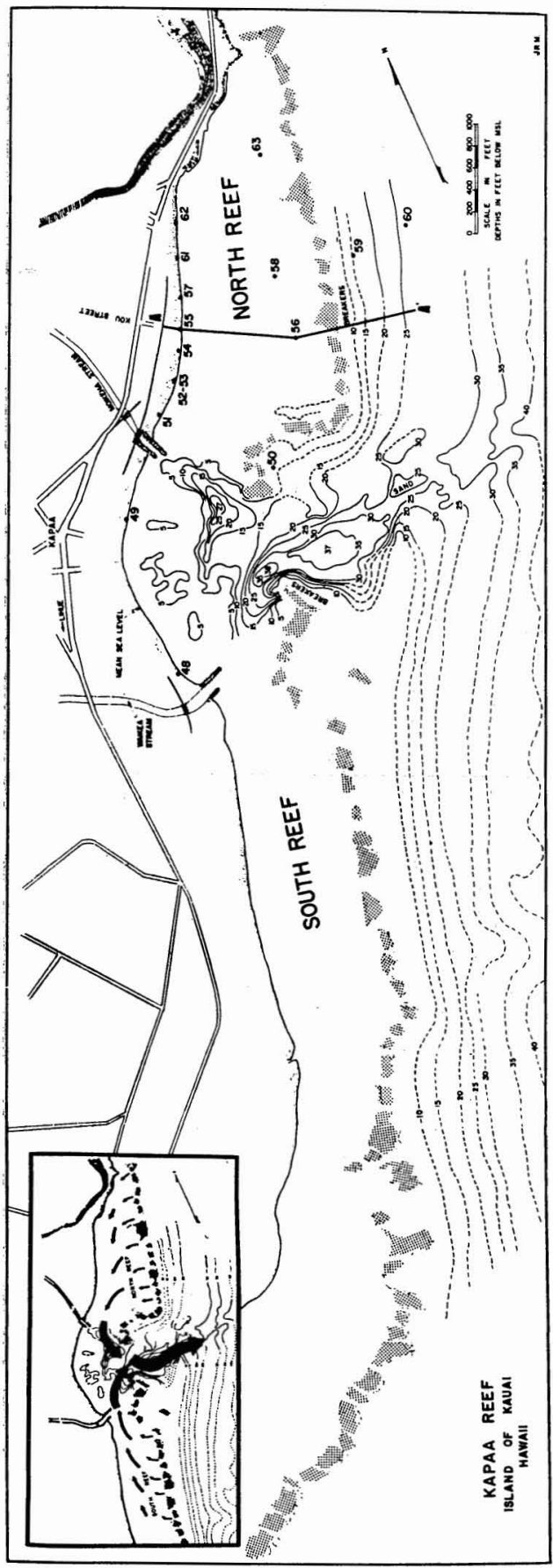

FIG. 8. Bathymetry and sample locations along $\mathrm{Ka}$ paa Reef. Dotted areas depict breaker zone on outer edge of reef platform. The circulation pattern of wave generated currents over the reef is shown in the inset. (Bathymetry compiled from Board of Harbor Com- in the rock results from a monomineralic species in the biogenous material of the original deposit. The presence of the single carbonate mineral species may be most easily accounted for by assuming (1) the inversion of an original aragonite component to calcite (see Jamieson, 1953); and (2) alteration of magnesium-rich to magnesium-poor calcite by the diagenetic loss of magnesium ions from an original magnesiumrich calcite component (see Chave, 1954b). The time required for such processes to be carried to completion, and the present physiographic position of this fossil dune, both suggest that it is considerably older than the neighboring beach rock.

\section{KAPAA-A WINDWARD REEF AND BEACH}

Kapaa Reef lies along the windward east coast of Kauai, and has an overall length of about $2 \frac{1}{2}$ miles along the north-south trending coastline in the vicinity of the town of Kapaa. It is a fringing reef and is divided into northern and southern portions by an inlet through the reef where two small streams enter the ocean near the center of the town. The Kealia River enters the ocean about 1 mile north of Kapaa, and the Wailua River enters the coastal embayment to the south of the reef. Although both rivers have appreciable runoff, they contribute relatively little sand-sized material to the littoral zone.

North Kapaa Reef is approximately $4,000 \mathrm{ft}$ long, and varies in width from about $400 \mathrm{ft}$ at its northern end to $1,500 \mathrm{ft}$ at the southern end, where it is terminated by the inlet (Fig. 8). The reef platform has an average depth of about $21 / 2 \mathrm{ft}$ below mean sea level, and the tide has a diurnal range of $1.8 \mathrm{ft}$. The inshore edge of the reef is bordered by a beach of medium- to coarse-sized calcareous sand. The beach has an average height of berm above mean sea level of about $61 / 2 \mathrm{ft}$, and has a total thickness of sand above the reef platform of about $8 \mathrm{ft}$. A remnant toe of beach rock, which outcrops intermittently on the reef flat 10-40 ft offshore from the present beach (Fig. 9), indicates a slight recession of the beach. The seaward portion of the

missioners Survey of 1919; photomosaic by R. M. Towell Inc., 1950; and miscellaneous soundings in 1957 (?) by H. V. Frasier, Hawaiian Dredging Co., and in 1959 by Sam Keala, Jr., of Lihue Plantation). 
reef is shallow, and causes waves to break in all weather. The seaward slope of the reef rises from a depth of about $15 \mathrm{ft}$, and is made irregular by numerous channels and solution and abrasion cavities (Figs. 9, 10). Beyond the base of the reef, the sea floor has a gentle slope out to the 60 - $\mathrm{ft}$ deep terrace. This terrace, which is common to the submarine topography of Kauai, occurs about 1 mile offshore in the vicinity of Kapaa. A sea-valley extends from near the inlet between North and South Kapaa Reefs, across the shelf and into deeper water (Figs. 2, 8).

The surface of the reef platform is very irregular and consists of a series of low intermittent channels and ridges (described by Munk and Sargent, 1954) which trend east-west and resemble in miniature the channels on the reef at Bikini Atoll. The channels are most pronounced on the northern and central portions of the reef and, although discontinuous, traverse the reef from the breaker zone to near the beach, where they enter the north-south trending depression which commonly exists at the toe of the beach.

\section{Littoral Processes on the Reef}

The circulation pattern of water over North Kapaa Reef is from north to south; over that portion of South Kapaa Reef near the inlet, it is from south to north (inset, Fig. 8). The currents flowing from the north and south reefs converge on the inlet and result in a seaward flow of several knots through the inlet. The anomalous depths of 27 and $38 \mathrm{ft}$, found relatively near shore in the inlet channel, are probably maintained free from sand by scour associated with the seaward-flowing current.

Measurements on North Kapaa Reef by Helfrich and Kohn (1957) show that both current velocity and the southerly component of velocity increase to the south. They obtained average values of current of about $1 \mathrm{ft}$ per second on the central portions of the reef and a maximum flow of about $11 / 2 \mathrm{ft}$ per second near the Moikeha Jetty. The most intense southerly flow of water occurs roughly one-third of the way between the beach and the seaward edge of the reef; currents at the toe of the beach were somewhat weaker.

The currents appear to result primarily from the mass transport of water associated with waves breaking over the reef. Winds may also be a factor in driving the currents, but their effect is minor, as evidenced by the prevailing northerly flowing current over portions of South Kapaa Reef. Also, on several occasions, southerly flowing currents were observed flowing over the north reef in opposition to brisk winds blowing from the south.

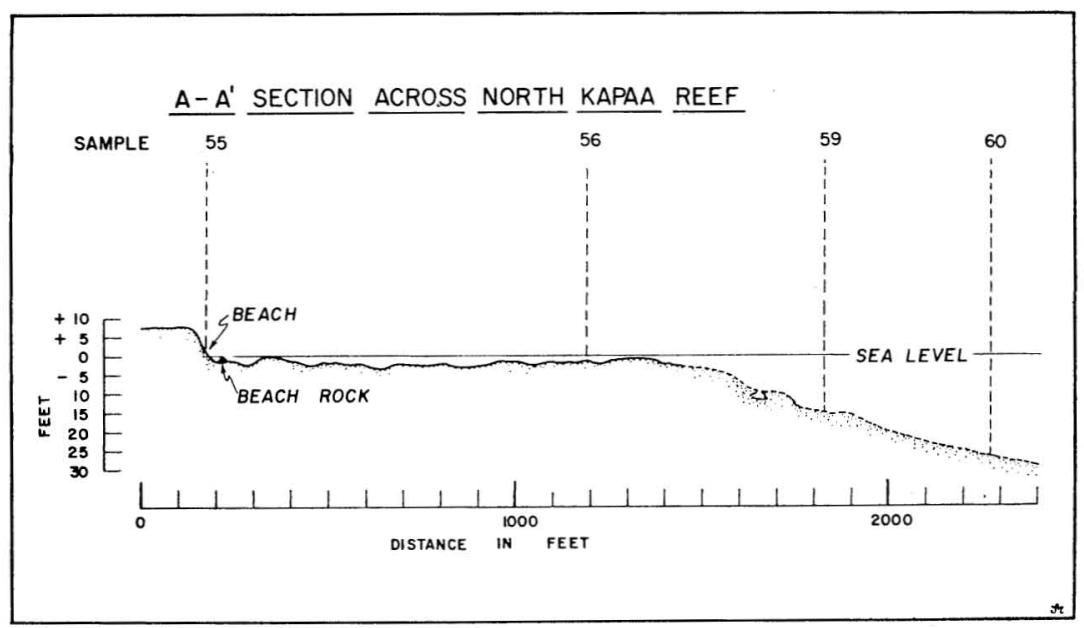

FIG. 9. Section across North Kapaa Reef along line shown on Figure 8. Vertical exaggeration is 10 x. Survey by Sam Keala, Jr., and Hiro Murashige of Lihue Plantation; outer reef and sea floor offshore from the reef based on soundings by authors. 


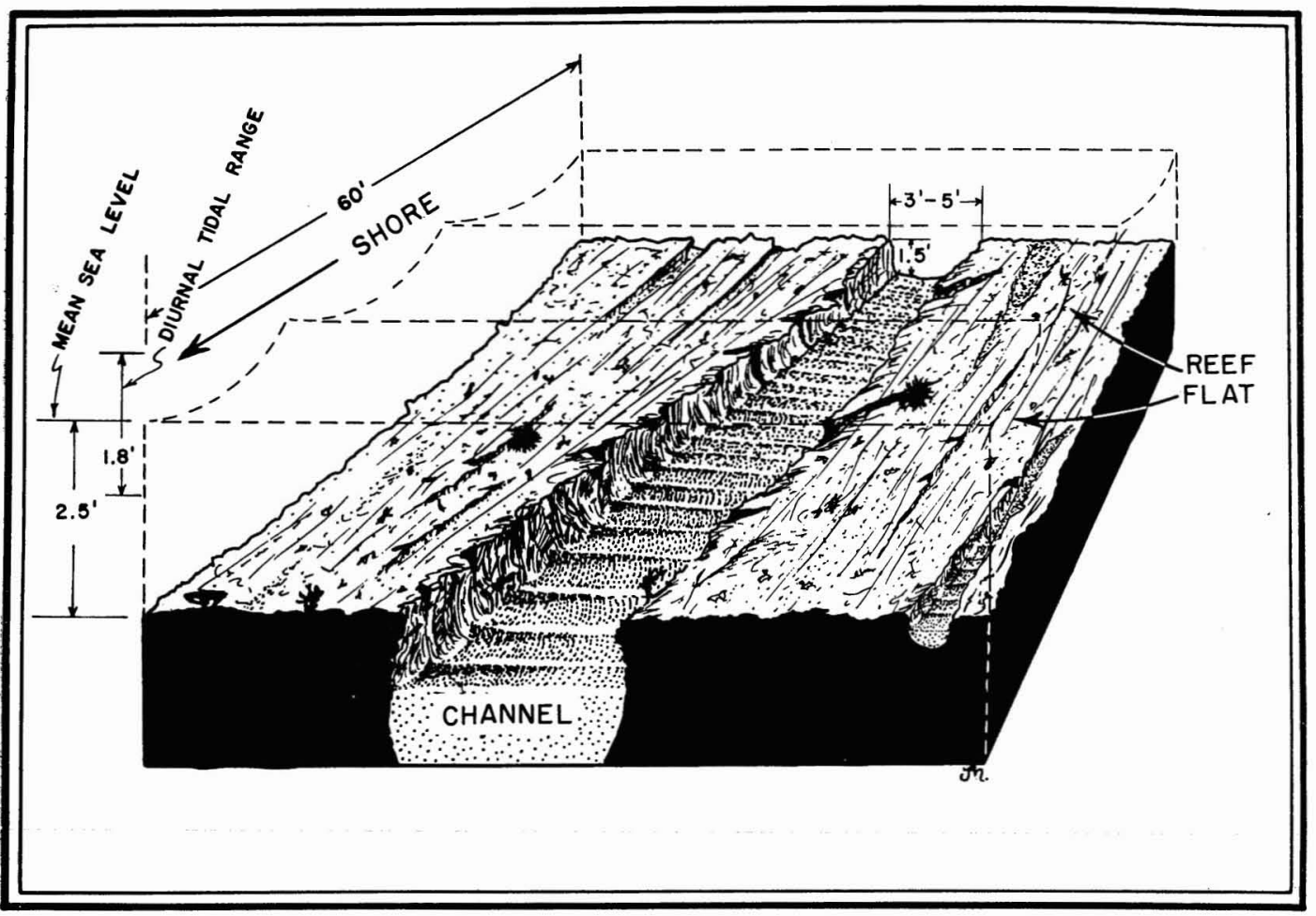

FIG. 10. Generalized sketch of surge channel on Kapaa Reef. Note sediment fill and ripples in bottom of channel. Sand in channels is moved shoreward by the small waves, which reform from the mass of water surging over the reef as larger waves break on outer reef. Prevailing currents, which flow from north to south (to the right of the viewer), are relatively ineffective in transporting sand on the reef flat.

Investigations indicate that the transportation of sand and the circulation of water over Kapaa Reef do not follow the same paths or cycles. While the water circulation is principally from north to south over the north reef, the east-west trending channels, which are common on the reef flat, act as traps for the sand, and prevent any appreciable southerly transport across the reef flat by water currents. The floors of the channels are covered with sand and reef rubble, in some places to a thickness of several feet. The sand is rippled by small waves moving across the reef flat from east to west, resulting in a net transport of sand toward the beach (Fig. 10). These low waves, which move sand across the reef flat, are formed from the mass of water which surges over the reef. The characteristic pattern is for waves of several feet or more in height to break on the outer reef, their momentum carrying a considerable amount of water over the reef in the form of a surge of white water. Over the reef flat, the surge first forms into a single wave crest, which may, in turn, disperse into a train of several smaller waves as the wave front moves toward the beach. The reef waves thus formed have heights in the neighborhood of $1 / 2$ to $1 \mathrm{ft}$, and wavelengths in the order of $20 \mathrm{ft}$. Water level and shoals on the reef control the height of the waves, for waves higher than the approximate depth of water tend to break and re-form as lower waves. Observations made while swimming over the reef indicate that the small waves are solitary in nature and that their particle or orbital velocity is greater in the onshore than in the offshore direction. This differential between onshore and offshore particle velocity, that accompanies the small waves, results in a step by step movement of sand toward the beach.

The east-west trending channels of the reef 
flat appear to play a unique role in the cycle of sand transportation over the reef. They function effectively as traps for sand that might otherwise be transported laterally along the reef by water currents and, at the same time, act as open conduits for the onshore transportation of sand by wave action.

Once on the beach, the sand probably undergoes a slow net southerly drift under the combined action of currents and waves impinging on the beach face. After migrating to the inlet, the sand is carried out to deeper water by the relatively strong currents that flow seaward through the inlet.

Thus, the transportation cycle of sand on the reef appears to include: (a) migration of sand along the channels in the reef flat, which is induced by wave action; (b) southerly transport of sand along the beach face and at the toe of the beach by waves and currents; and (c) seaward transport of sand through the inlet and into deeper water by the currents which converge from the north and south reef and flow seaward.

The sand on the beaches apparently is derived from organisms living on the reef, and from material transported in suspension by waves breaking over the reef. Diving observations indicated that the numerous solution cavities and the general rough outer edges of the reef cause considerable turbulence in the breaking and nearbreaking waves. This turbulence increases their capacity for carrying material in suspension. Undoubtedly some reef material also undergoes a net offshore migration by processes of turbulent diffusion. Whether this loss is greater or less than the supply of material to the reef from offshore is not known.

The position and growth of the reef, in recent geologic time, appears to be governed by the combined effects of land runoff and the water circulation and sand transportation over the reef system. Inlets tend to occur in the fringing reefs where streams enter and bring fresh water and mud, which are detrimental to the reef building corals and algae. The reef builders flourish where wave action is most vigorous. Therefore, the reef platform becomes more extensive in the areas not influenced by land runoff. The formation of a wider reef platform causes more and more water and sand to cross the reef and flow into the inlets. The scouring action on the inlets by water and sand derived from the reef maintains the inlets even when rivers are not flowing. Thus it would appear that once the position of a potential inlet is determined by land runoff and coastal configuration, the pattern of water circulation and sand transportation on the growing reef will tend to perpetuate the location of the inlet.

\section{Kapaa Sediments}

Samples collected from the beach, reef flat, and sea floor outside the reef all consisted of very coarse, coarse, and medium-grain sands, containing about $90 \%$ calcium carbonate of biogenous origin. The following trends in the sediment were observed from north to south along the north Kapaa reef flat: (1) the sand becomes finer, (2) the sands become better sorted, (3) the magnesium content of the calcite decreases, and (4) the foraminifera content in the sand decreases. In general, the reef flat and offshore samples were coarser than the beach samples. These trends, although not necessarily definitive in themselves, are in agreement with the modes and cycles of sediment transport as discussed previously. The samples from the Kapaa area contained a higher percentage of magnesium-poor calcite than any of the other unconsolidated sediment samples collected from Kauai. In general, the content of magnesium-poor calcite increases southward on Kapaa beach and seaward across the reef. The distribution of magnesium-poor calcite is consistent with the supposition that the supply of beach sand is predominantly from transportation over the reef opposite the beach, rather than from transportation along the shore.

The only completely identifiable biogenous. components present in significant quantities in sands were the foraminifera Heterostegina suborbicularis and Amphistegina madagascariensis. A rough estimate indicated that these species made up $3 \%$ to $20 \%$ of the grains in the samples. All of the tests were considerably worn, making it quite difficult to separate the two species under a microscope. A more accurate determination of their relative abundance, based on their skeletal composition, was obtained by $\mathrm{X}$-ray diffraction. In sample 59 , about $55 \%$ of the foraminiferal carbonate consisted of magnesium-rich calcite, indicating deposition by Het- 


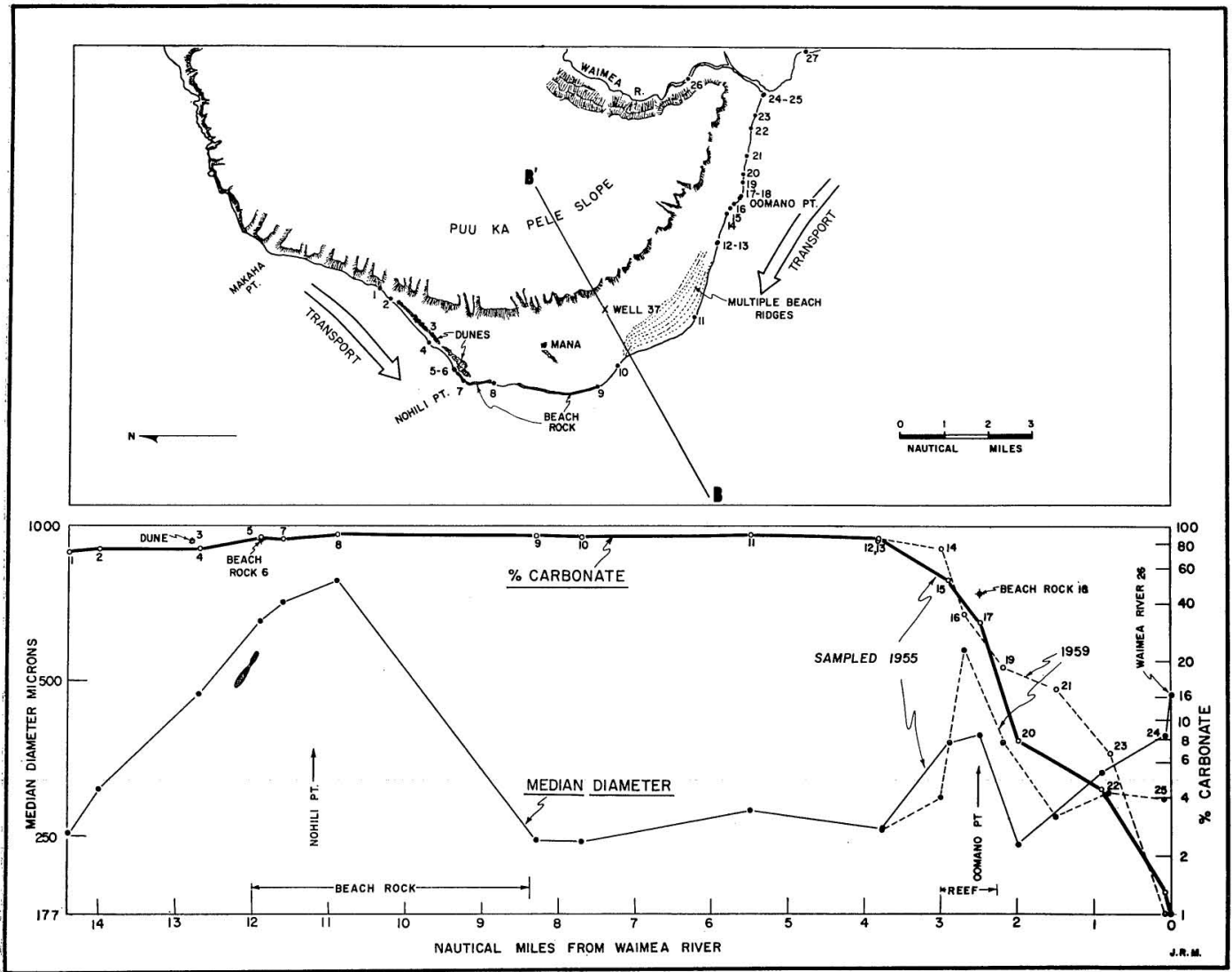

FIG. 11. Top: Direction of littoral transport and sample locations along the Mana Coastal Plain. Note beach rock outcrops, and position of multiple beach ridges and recent and ancient dunes.

Bottom: Variation in carbonate content and median grain size with distance along the Mana shoreline. Median diameters determined by Emery settling tube.

erostegina. The remaining $45 \%$ was magnesiumpoor calcite and presumably is derived from Ampbistegina. Very little is known of the living depth range of either species. The highly worn appearance of all of the tests suggest that on Kauai they do not live on the reef but have been transported some distance. ${ }^{7}$

\section{MANA COAST-LEEWARD BEACHES}

The Mana Coastal Plain (Fig. 11) consists of calcareous beach, dune, and lagoon sediments, mainly of marine biogenous origin, but including some terrigenous sands near the Waimea

${ }^{7}$ Ruth Todd, U. S. Geological Survey, suggests that both species may thrive in water down to at least 200 $\mathrm{ft}$ (personal communication).
River. Well borings show that these marine sediments are about $125 \mathrm{ft}$ thick near the base of the Puu Ka Pele slope, and may be $400-500 \mathrm{ft}$ thick at the seaward edge of the Mana Coastal Plain. They lie on a gently sloping bench, apparently cut by waves into the original dome surface during a lower stand of sea level (Fig. 12 ).

The region west of the Waimea River, consisting of the Mana Coastal Plain and the Puu $\mathrm{Ka}$ Pele slope, is practically a rainshadow desert. This region receives an average annual rainfall of 18 inches (Table 1), the lowest rainfall of any major drainage area on the island. The lee coast has few fringing reefs, and those that do occur are more poorly developed than the reefs on the exposed windward coast. 
Before man changed the drainage in this area, most of the water that drained off of the Puu $\mathrm{Ka}$ Pele slope or that was discharged by springs from the ground-water body beneath the slope was ponded by the coastal sand dunes, forming a fresh to brackish marsh. Now even though the marsh has been drained, it seems unlikely that significant amounts of sediment are being supplied to the shore by streams other than the Waimea River. The presence of large calcareous dunes and the dominant marine nature of the sediments of the Mana Coastal Plain indicate the importance of the transport of biogenous material from the sea to the land. Prograding of the shoreline of the Mana Coastal Plain, during the recent geologic past, is indicated by the occurrence of multiple beach ridges west of Oomano Point, and at Mana by the lithified dunes situated two miles inland and parallel to the present coastal dunes at Nohili Point.

The carbonate content of the samples was high everywhere except near the Waimea River, where the sediments were principally terrigenous. The carbonate content increased towards the center of the Mana Coastal Plain, attaining a maximum value of about $90 \%$ at sample stations 8 and 9 (Fig. 11, top). The median diameter of the samples ranged from about 250 to $800 \mathrm{mi}$ crons, with those near Nohili and Oomano Point being significantly coarser, probably as a result of increased wave action on the points.
There was a pronounced bimodality in the size distribution, both by sieving and settling tube, of sediments from stations 9 and 10. This seems to reflect the mixing of sediments from the Napali-Nohili region with those from the south coast. The major mode occurs at $180 \mathrm{mi}$ crons, with a minor near 700 microns (Fig. 5). Microscopic examination shows the major mode to consist of unidentifiable fine, angular, carbonate grains; the principal constituent of the coarse mode was foraminifera, mostly Heterostegina suborbicularis. In comparison, sample 15 , which consists of approximately equal portions of terrigenous and biogenous material, is better sorted and shows less tendency towards bimodality. Sample 15, which was collected nearly 3 miles west of Waimea River, is from a region of active littoral transport, where the rate of sorting approaches the rate of mixing of unlike materials. Carbonate content and size distribution of the sediments indicate that the terrigenous material is transported along the beach westward from the mouth of the Waimea River and southward from the Napali Coast, and that as it is transported it becomes progressively more diluted by biogenous material. The logarithm of the carbonate content is observed to increase linearly with distance from the Waimea River and from the Napali Coast (Fig. 11, bottom).

The increase in carbonate content with distance from the terrigenous source is indicative

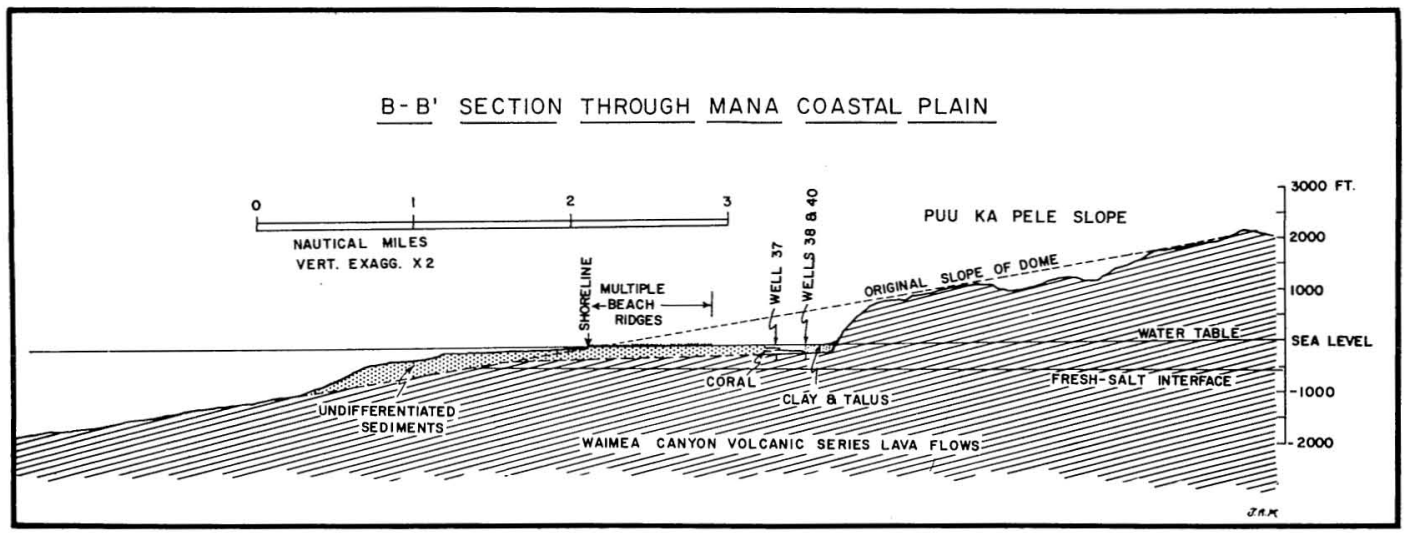

FIG. 12. Section through Mana Coastal Plain (along line shown in Fig. 11). Well numbers and well data taken from Macdonald, Davis, and Cox (1960). Composition of the submarine portion of the profile is little known and is based principally on the supposition that the section above the line of original slope of the dome is undifferentiated sediments, probably deposited by littoral currents at a lower sea level. 
of continuous dilution by the addition of biogenous material. An expression giving the dilution rate can be derived under the following assumptions: (1) sand transported along the shore consists of a mixture of biogenous and terrigenous material, where the fraction of each is $B$ and 1-B respectively; (2) this sand is transported along the shore at a rate $\mathrm{Q}$ which is constant with distance $\mathrm{x}$ along the coast; (3) biogenous material is supplied to the beach, from offshore, at a constant rate $F_{i}$ per unit of coast length, where it is completely mixed with the sand transported along the shore; and (4) there is a net deposition of this mixed sediment at a rate $F_{o}$ per unit of coast length, such that $F_{i}=F_{o}$.

Conditions 1 through 4 lead to the following general expression for the concentration of biogenous material in the beach sand as a function of the longshore transport rate $Q$, the rate of supply of biogenous material $F_{i}$, and distance along the coast $\mathrm{x}$ :

$$
\ln B=\frac{F_{i}}{Q} x+\text { constant of integration }
$$

where $1 \mathrm{n} B$ is the natural logarithm of the carbonate fraction (see Appendix I). This is a straight line with slope $F_{i} / Q$ when $B$ is plotted on a logarithmic and $\mathrm{x}$ on a linear scale (Fig. 11 , bottom). If the terrigenous material is supplied to the coast in large quantities and at a single point, as at the mouth of the Waimea River, B will be almost zero near the river and will increase exponentially with distance from the river-as it is observed to do.

The relation derived here is useful in that it not only predicts the form of the concentration change, but also indicates that, if either the longshore transport of sediment $\mathrm{Q}$, or the deposition rate $\mathbf{F}$ is known, the other can be computed. The equation for the curve of concentration change with distance from the Waimea River is:

$$
\text { ln } B=1.4 x-4.6
$$

where $1 \mathrm{n} \mathrm{B}$ is the natural logarithm of the carbonate fraction, $x$ is in nautical miles, and $F_{i} / Q=1.4$ has the units of nautical miles ${ }^{-1}$.

Neither $F_{1}$ nor $Q$ are known, but very rough order-of-magnitude estimations can be made for both. The rate of accumulation of calcareous sediment on the Mana Coastal Plain, divided by the length of coast line, gives a measure of $F_{i}$, while the sediment yield from the Waimea River drainage basin provides an estimate for the littoral transport rate $Q$ near the mouth of the Waimea River.

The volume of sediment in the Mana Coastal Plain down to a depth of $60 \mathrm{ft}$ below sea level is approximately $4 \times 10^{10} \mathrm{ft}^{3}$. If it is assumed that $60 \%$ of this is of biogenous origin, and that it has accumulated along a coastal length of 15 nautical miles during a time interval of 8,500 years $^{8}$, then the rate of supply of biogenous material to the coast, $F_{i}$, is about 7000 cubic yd per mile per year. This gives a value for the littoral transport rate in the Waimea region of:

$$
\mathrm{Q}=\mathrm{F}_{\mathrm{i}} / 1.4=5,000 \mathrm{yd}^{3} \text { per year. }
$$

The Waimea drainage basin, with an average effective precipitation of 53 inches per year (Table 1), would be expected to yield about 420 tons of sediment per square statute mile per year (Langbein and Schumm, 1958: fig. 3). Assuming half of this to be sand-size or larger material, and using the conversion from weight to volume of $60 \mathrm{lbs}$ per $\mathrm{ft}^{3}$ (given by Langbein and Schumm, 1958), one obtains a total yield of sand-size material from the Waimea basin of $25,000 \mathrm{yd}^{3}$ per year. Since the Waimea River does not have a prograding delta, the yield from the river must nearly equal the littoral transport rate $\mathrm{Q}$.

Beach surveys of 1926 and 1950 by the Corps of Engineers (1955: par. 52) indicated a net loss of sand from Waimea Beach of 5,000 $\mathrm{yd}^{3}$ per year. The littoral transport rate is probably greater than the loss, and the Corps of Engineers estimate it to be about $20,000 \mathrm{yd}^{3}$ per year. Considering the nature of the assumptions leading to the calculations of littoral transport rate in the preceding paragraphs, values of 5,000 and $25,000 \mathrm{yd}^{3}$ per year are remarkably, if not fortuitously, similar. One must recognize that, in so far as the actual transport rates at Waimea are concerned, these calculations may eventually prove to be merely a mental exercise. If this be the case, it is hoped that they have at least served to illustrate a valid principle.

\footnotetext{
${ }^{8}$ The rate of $60 \mathrm{ft}$ sea level rise in 8,500 years was selected because this seems to be the most accurate and significant part on the sea level rise curves, presented by Shepard (1960: fig. 4), McFarlen (1961: fig. 9), and Jalgersma and Pannekoek (1960: fig. 3).
} 


\section{ACKNOWLEDGMENTS}

This paper represents, in part, results of research carried out by the University of California under contract with the Office of Naval Research. The original field work was performed in November and December 1955. Supplementary field study in January 1959 was supported by the Lihue Plantation Company, Kauai. Valuable advice and field assistance during the supplementary study was given by J. T. Orrick and S. L. Keala, Jr., of the Lihue Plantation. Since this study, North Kapaa Reef has been modified by dredging. Unfortunately the dredging was not performed entirely in accordance with plans based on the study of the littoral processes over the reef as outlined here. A north-south channel was dredged, which may intercept the sand supply to the beaches and cause the beach to erode. ${ }^{9}$

Valuable suggestions and guidance during the course of the study were contributed by R. S. Arthur, E. D. Goldberg, F. P. Shepard, E. W. Fager, and M. N. Bramlette of the University of Califoria. Suggestions leading to the section on exposure to waves and wind were made by P. L. Horrer of Marine Advisers, La Jolla. Identification of foraminifera was made by Ruth Todd of the U. S. Geological Survey, Washington. In addition, the writers wish to express their appreciation to H. S. Ladd and J. I. Tracey, Jr., also of the U. S. Geological Survey, for their careful reading of the manuscript, and to Edith Haselwood of the Hawaiian Sugar Planters' Association for her editorial criticism.

\section{APPENDIX I}

\section{DERIVATION OF SEDIMENT TRANSPORT EQUATION}

A small segment of shoreline (see figure) is assumed to be in a state of equilibrium, such that the rate of longshore transport of material into the segment (the longshore influx) is constant and equal to the outflux, $Q_{i}=Q_{0}$. Also, the onshore influx and outflux are constant and equal, $F_{i}=F_{0}$. The sediment influx from offshore, $\mathrm{F}_{\mathrm{i}}$, consists of $100 \%$ biogenous material,

\footnotetext{
${ }^{8}$ Comparison of surveys of 1959 and 1962 indicate a net erosion of beach immediately westward of the dredging area (approximately stations 51-57, Fig. 8) of about 2,500 cubic yd per year.
}

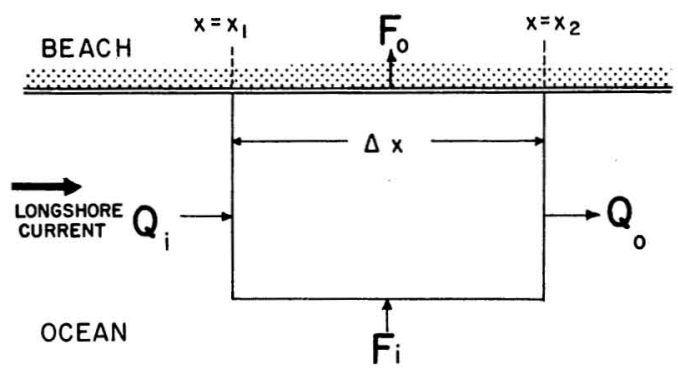

$B$; while the sediment $Q_{i}$ transported longshore consists of two components, a terrigenous fraction $\mathrm{T}$, and a biogenous fraction $\mathrm{B}=1-\mathrm{T}$. If complete mixing takes place within a coastal segment, having boundaries $\mathrm{x}_{1}$ and $\mathrm{x}_{2}$, a distance $\triangle \mathrm{x}$ apart, then $\mathrm{T}$ is a function of the longshore distance $\mathrm{x}$ and can be written $\mathrm{T}(\mathrm{x})$. The above assumptions permit the budget for the fraction of terrigenous material to be written:

$$
\begin{gathered}
\mathrm{Q} \cdot \mathrm{T}\left(\mathrm{x}_{1}\right)=\mathrm{Q} \cdot \mathrm{T}\left(\mathrm{x}_{2}\right)+\mathrm{F}_{\mathrm{o}} \mathrm{T}\left(\mathrm{x}_{1}+\frac{\Delta \mathrm{x}}{2}\right) \Delta \mathrm{x} \\
\mathrm{O}=\frac{\mathrm{Q} \mathrm{T}\left(\mathrm{x}_{1}\right)-\mathrm{T}\left(\mathrm{x}_{2}\right)}{\Delta \mathrm{x}}+\mathrm{F}_{\mathrm{o}} \mathrm{T}\left(\mathrm{x}_{1}+\frac{\Delta \mathrm{x}}{2}\right)
\end{gathered}
$$

but in the limit as $\Delta \mathrm{x} \longrightarrow \mathrm{o}$

$$
\begin{gathered}
\mathrm{o}=\mathrm{Q} \frac{\mathrm{dT}}{\mathrm{dx}}+\mathrm{F}_{\mathrm{o}} \mathrm{T}(\mathrm{x}) \\
\frac{\mathrm{dT}}{\mathrm{T}}=-\frac{\mathrm{F}_{\mathrm{o}}}{\mathrm{Q}} \mathrm{dx}
\end{gathered}
$$

and integration gives $\ln \mathrm{T}=-\frac{\mathrm{F}_{0}}{\mathrm{Q}} \mathrm{x}+\mathrm{k}$

where $\ln$ is the base of natural logarithms and $\mathrm{k}$ is a constant of integration. The relation may be written in terms of $\mathrm{B}=1-\mathrm{T}$ as:

$$
\ln B=\frac{F}{Q} x+k
$$

\section{REFERENCES}

ARTHUR, R. S. 1948. Forecasting Hawaiian swell from January 2 to 5, 1947. Bull. Amer. Meteor. Soc. 29:395-400.

BIEN, G. S. 1952. Chemical analysis methods. Scripps Inst. Oceanogr. Ref. 52-58. 
Blackmon, P. D., and R. TodD. 1959. Mineralogy of some foraminifera as related to their classification and ecology. J. Paleo. 33 (1):115.

Bramlette, M. N. 1926. Some marine bottom samples from Pago Pago Harbor, Samoa. Carnegie Inst. Publ. 344.35 pp.

Chave, K. E. 1954a. Aspects of the biogeochemistry of magnesium 1, calcareous marine organisms. J. Geol. 62:266-283.

1954b. Aspects of the biogeochemistry of magnesium 2, calcareous sediment and rocks. J. Geol. 62:587-599.

Corps of EngIneers, San Francisco District. 1955. Beach erosion control report on cooperative study of Waimea Beach in Hanapepe Bay, Island of Kauai, T. H. (Partially published as House Doc. 432, 84th Cong. 2nd Sess.)

Emery, K. O., and D. C. Cox. 1956. Beach rock in the Hawaiian Islands. Pacific Sci. 10 (4): 382-402.

GAYMAN, W. R. In preparation. X-ray diffraction calibration curves for ratios of aragonite and magnesium-rich and magnesium-poor calcite.

GOLDSMith, J. R., D. L. GRAF, and O. I. JoENsuU. 1955. The occurrence of magnesium calcites in nature. Geochim. Cosmochim. Acta. $7: 212-230$.

Hinds, N. E. A. 1930. Geology of Kauai and Niihau. Bishop Museum Bull. 71.

INMAN, D. L. 1949. Sorting of sediments in the light of fluid mechanics. J. Sed. Petrol. 19:5170 .

1952. Measures for describing the size distribution of sediments. J. Sed. Petrol. 22: 125-145.

1953. Areal and seasonal variations in beach and nearshore sediments at La Jolla, California. Beach Erosion Board, Corps of Engineers, Tech. Memo. 39. 134 pp.

Jamieson, J. C. 1953. Phase equilibrium in the system calcite: aragonite. J. Chem. Physics 21:1385-1390.
Kohn, A. J., and P. Helfrich. 1957. Primary organic productivity of a Hawaiian coral reef. Limnology and Oceanography 2:241-251.

Jelgersma, S., and A. J. PANnekoek. 1960. Post-glacial rise of sea-level in the Netherlands. Geol. Mijnbouw 39:201-207.

LANGBeIN, B. et al. 1949. Annual runoff in the United States. U. S. Geol. Surv. Circ. 52. 14 pp.

and S. A. Schumm. 1958. Yield of sediment in relation to mean annual precipitation. Trans. Amer. Geoph. Union 39:10761084

Lowenstam, H. A. 1954. Factors affecting the aragonite-calcite ratios in carbonate secreting marine organisms. J. Geol. 62:284-322.

Macdonald, G. A., D. A. Davis, and D. C. Cox. 1960. Geology and ground water resources of the Island of Kauai, Hawaii. Haw. Div. Hydrog. Bull. 13. 212 pp.

MCFARLAN, E., Jr. 1961. Radiocarbon dating of Late Quaternary deposits, South Louisiana. Geol. Soc. Amer. Bull. 72:129-158.

Munk, W. H. and M. C. SARgent. 1954. Adjustment of Bikini Atoll to ocean waves. U. S. Geol. Surv. Prof. Paper 260-C:275-280.

Poole, D. M., W. S. Butcher, and R. L. FISHER. 1951. The use and accuracy of the Emery settling tube for sand analysis. Beach Erosion Board, Corps of Engineers Tech. Memo. 23. 11 pp.

Shepard, F. P. 1960. Rise of sea level along northwest Gulf of Mexico. Recent Sediments, Northwest Gulf of Mexico, 1951-1958. Amer. Assoc. Petr. Geol. 1960:338-381.

, G. A. Macdonald, and D. C. Cox. 1950. The tsunami of April 1, 1946. Bull. Scripps Inst. Oceanogr. 5:391-528.

Territorial PlanNing BoARd, Territory OF HAwAII. 1939. Surface water resources of the Territory of Hawaii, 1901-1938; Summary of Records. Honolulu Star-Bulletin Limited. $411 \mathrm{pp}$. 